\title{
Thermodynamically-stable two-phase
}

\section{equilibrium calculation of hydrocarbon}

\section{mixtures with capillary pressure}

\author{
Yiteng $\mathrm{Li}^{\dagger}{ }^{\dagger}$ Jisheng Kou, ${ }^{\ddagger}$ and Shuyu Sun ${ }^{*, \dagger}$ \\ $\dagger$ Physical Science and Engineering Division (PSE), King Abdullah University of Science \\ and Technology (KAUST), Thuwal, 23955-6900, Saudi Arabia \\ $\ddagger$ School of Mathematics and Statistics, Hubei Engineering University, Xiaogan, 432000, \\ Hubei, China \\ E-mail: shuyu.sun@kaust.edu.sa
}

\begin{abstract}
With conventional reservoirs being depleted, unconventional reservoirs play a significant role in worldwide energy supply. Even though the exploitation of unconventional resources has achieved unprecedentedly great success, flow mechanisms and the underneath phase behavior are not clearly illustrated. Recent studies indicate that the confined space in nanoscale and the considerable capillary pressure result into a significant deviation of fluid properties from their bulk properties. Failure to incorporate these effects will lead to inaccurate estimation of reservoir performance. As a consequence, the confinement effect and capillary pressure should be taken into account to accurately characterize the phase behavior in unconventional reservoirs. In this study, capillary effect is incorporated into phase equilibrium calculations at constant moles, volume and temperature which is believed to have advantages over phase equilibrium calculations at
\end{abstract}


constant moles, pressure and temperature in many cases. We develop evolution equations for moles and volume using the laws of thermodynamics and Onsager's principle. A thermodynamically stable numerical algorithm is constructed, which is the extension of our previous work, by introducing a generalized Onsager coefficient matrix. The new algorithm exhibits better performance in efficiency and accuracy by simultaneously solving the evolutionary equations. At the equilibrium state, the pressure inequality resulting from the capillary pressure is spontaneously satisfied. In addition, by properly selecting the initial approximation for phase equilibrium calculations, we obtain a physically meaningful transition process with uniform phase identification. A number of numerical examples is presented to demonstrate the robust performance of the proposed model. It is found that capillary pressure affects phase composition and distribution. The bubble point pressure is suppressed under capillary effect, while the dew point pressure exhibits complex behavior: increasing in the upper branch and decreasing in the lower branch of the dew point curve. The unremarkable saturation change may attribute to the volume variation less sensitive than the pressure variation when the phase composition changes. In the limit of zero capillarity, our simulation results are consistent with the conventional isothermal-isochoric flash calculation.

\section{Introduction}

In the past decade, due to the technology of multistage hydraulic fracturing and horizontal well, the production of unconventional resources, including shale gas and tight oil, has had a blowout-growth in North America and now takes a place in the world's energy structure. Despite of this, the evaluation, development and management of unconventional reservoirs still rely on accurate reservoir simulation. In conventional approaches, capillary effect is often ignored in phase equilibrium calculation as if the individual pore serves as a PVT

cell. However, the presence of nanopores in shale and tight-rock formations ${ }^{1-3}$ yields a large capillary pressure and confinement effect. ${ }^{4-7}$ As a result, the confined fluid properties 
deviate from their bulk properties. Such variations significantly impact well performance and ultimate recovery of unconventional reservoirs. ${ }^{8}$ Thus, it is necessary to incorporate capillary pressure into phase equilibrium calculation for reliable modeling phase behavior in shale and tight reservoirs.

There are a number of studies in literature investigating the effect of capillary pressure on the thermodynamic behavior of hydrocarbon mixtures. Brusilovsky ${ }^{9}$ simulated the vapor/liquid equilibrium (VLE) of reservoir fluid in the presence of capillary pressure by a general cubic equation of state (EOS). Nojabaei et al. ${ }^{10}$ investigated the confined phase behavior of simple binary mixtures and real Bakken shale oil over a wide range of pressure and temperature using the Peng-Robinson EOS. ${ }^{11}$ Sandoval et al. ${ }^{12}$ proposed an algorithm to construct the phase envelope of multicomponent and reservoir fluid systems under the effect of capillarity, however, with the Soave-Redlich-Kwong EOS. It should be noted that molecule-surface interactions are ignored in the preceding studies. The negligible confinement effect claims the pore size should be no less than $10 \mathrm{~nm}$. However, fluid properties in the nanopores less than $10 \mathrm{~nm}$ have been discovered to significantly deviate from their bulk values, due to the increasing interaction between fluid molecules and pore walls. In order to characterize phase behavior in the confined space of smaller pore size, Jin et al. ${ }^{13}$ and Teklu et al. ${ }^{14}$ incorporated critical temperature and pressure shifts to account for the nanoscale confinement. Both studies have shown the shift of critical properties plays a significant role in accurate prediction of phase behavior in the pores of a few nanometer. In addition, some authors combined the compositional simulation with the confined phase equilibrium calculation to investigate the effect of capillary pressure on the performance of unconventional reservoirs either with or without fractures. ${ }^{15-17}$ It was pointed out that failure to consider the capillary equilibrium problem leads to inaccurate estimation of original oil in place and ultimate recovery. Furthermore, instead of using the Young-Laplace equation, Wang et al. ${ }^{16}$ and Rezaveisi et al. ${ }^{17}$ suggested to apply the Leverett J-function to evaluate the capillary pressure in porous media. However, since we place more importance on phase equilibrium 
calculation itself rather than the simulation of subsurface flow, the Young-Laplace equation is used to compute capillary pressure in this work.

All the aforementioned literature deals with the VLE problems in which the vapor pressure differs from the liquid pressure. Such a problem is a modification of the conventional flash calculation at constant pressure and temperature (the so-called PT-flash). Compared to the unconfined PT-flash problem, an additional pressure equilibrium condition is required to enforce the pressure inequality caused by capillary pressure. Additionally, the chemical equilibrium condition is modified to take into account the capillary effect. Although pressure and temperature are the most commonly used specification variables for phase equilibria problems, the limitations of the PT-formulation have been discussed in refs. 18-20. Apart from computational inefficiency, the realistic equation of state formulated by pressure and temperature has nonunique solutions as it is inevitable to solve a cubic equation. Another drawback is that the volume of a pure substance at the saturation pressure is ambiguous. Similar observations have been made for the multicomponent mixtures with three or four phases. Due to the above limitations, phase equilibrium calculation at the given volume and temperature, known as VT-flash, has drawn increasing attention in the recent years. Among them, Jindrová and Mikyška ${ }^{19,20}$ developed fast and robust algorithms for two-phase and multiphase equilibrium calculation at constant moles, volume and temperature by direct minimization of the total Helmholtz free energy. Kou et al. ${ }^{21}$ proposed a dynamic model and an energy-stable algorithm for VT-flash problems. The evolution equations for mole numbers and volume are established on the basis of the Fick diffusion and mechanical mechanism. Cabral et al. ${ }^{22}$ formulated the phase equilibrium problem in the presence of adsorption and solved it by minimizing the Helmholtz free energy. Smejkal and Mikyška ${ }^{23}$ presented a general formulation for PT-, VT- and UV-flash (phase equilibrium calculation at constant internal energy and volume) problems. A numerical algorithm was developed by directly minimizing the objective function under constraints. They compared the numerical performance of different formulations and concluded that the performance of the VT-flash 
calculation is identical or better than that of the PT-flash calculation.

Regardless of what the variable specification is, phase stability testing is always applied in conjunction with the phase splitting algorithm for flash calculation. Stability analysis is used to decide whether a fluid mixture is stable or not at the given condition. A variety of methods have been developed to locate the global minimum of the tangent plane distance (TPD) function either by direct search or by the local minimization using multiple guesses. If a trial phase is found such that the value of TPD function is negative, the mixture is unstable and phase split will occur. The trial phase composition will be used to initialize phase equilibrium calculation. Various implementations of VT-stability testing can be found in refs. ${ }^{24-29}$

The above VT-flash problems are all defined in unconfined space. To the best of our knowledge, the investigation to the confined VT-flash problem is rare. In this study, capillary pressure is incorporated into the phase equilibrium calculation of two-phase systems at specified moles, volume and temperature. We ignore both confinement effect and adsorption and use a single pore size as most of the studies on capillary equilibrium do. The evolution equations are formulated using the laws of thermodynamics and Onsager's reciprocal principle. A thermodynamically stable numerical algorithm is extended from our previous work ${ }^{30}$ by introducing a generalized Onsager coefficient matrix which is computed based upon the unconfined equilibrium conditions. The new algorithm simultaneously solves the evolutionary equations and exhibits better performance in efficiency and accuracy. Adaptive time step is applied to improve the computational efficiency further. At the equilibrium state, the pressure inequality resulting from the capillary pressure is spontaneously satisfied. We obtain a physically meaningful transition process with uniform phase identification by properly selecting the initial approximation for phase equilibrium calculations. The applicability of the proposed model and algorithm is tested from single-component to multicomponent fluid either with and without capillary pressure.

The rest of this paper is organized as follows. In the next section, we briefly introduce 
the Helmholtz free energy of the isothermal-isochoric system. In section 2 and 3, we formulate the work done by the capillary force and the evolution equations for moles and volume. The thermodynamically stable numerical algorithm is presented in section 4 , with the generalized Onsager coefficient matrix. The numerical simulation is outline in section 5 . We present a number of numerical examples and discuss the simulation results in the Results and Discussion. At the end, concluding remarks are made.

\section{Helmholtz free energy of the isothermal-isochoric system}

Consider a fluid mixture of $M$ components occupies volume $V$ at temperature $T$. The system will split into two phases if it is unstable at the given condition. We denote by $f(\mathbf{n})$ the Helmholtz free energy density which has the following form

$$
f(\mathbf{n})=f^{\text {ideal }}(\mathbf{n})+f^{\text {repulsion }}(\mathbf{n})+f^{\text {attraction }}(\mathbf{n})
$$

with

$$
\begin{aligned}
f^{\text {ideal }}(\mathbf{n}) & =R T \sum_{i=1}^{M} n_{i}\left(\ln n_{i}-1\right) \\
f^{\text {repulsion }}(\mathbf{n}) & =-n R T \ln (1-b n) \\
f^{\text {attraction }}(\mathbf{n}) & =\frac{a(T) n}{2 \sqrt{2} b} \ln \left(\frac{1+(1-\sqrt{2}) b n}{1+(1+\sqrt{2}) b n}\right)
\end{aligned}
$$

where $n=\sum_{i} n_{i}$ is the overall molar density. Thus, the Helmholtz free energy $F(\mathbf{n})$ of a two phase system is formulated as

$$
F=f\left(\mathbf{n}_{1}\right) V_{1}+f\left(\mathbf{n}_{2}\right) V_{2}
$$

where $\mathbf{n}_{\alpha}=\mathbf{N}_{\alpha} / V_{\alpha}, \alpha=1,2$. Let $\mathbf{n}_{\alpha}=\left[n_{1, \alpha}, \ldots, n_{M, \alpha}\right]^{T}, \mathbf{N}_{\alpha}=\left[N_{1, \alpha}, \ldots, N_{M, \alpha}\right]^{T}$ and $V_{\alpha}$ represent the molar density, mole number and volume of phase $\alpha$, respectively. For VT-flash 
problems, the mole constraint $\mathbf{N}_{1}+\mathbf{N}_{2}=\mathbf{N}$ and the volume constraint $V_{1}+V_{2}=V$ must be satisfied.

\section{Work of capillary force}

In order to incorporate capillary effect into our formulation, the work done by the capillary pressure needs to be quantified. We assume two phases are separated by a curved interface of zero thickness. Let $p_{1}$ and $p_{2}$ represent the pressure of phase 1 (nonwetting) and phase 2 (wetting). The capillary pressure can expressed as $p_{c}=p_{1}-p_{2}$. When capillary force does positive work, the interface moves toward phase 1 so that the volume of phase 1 is compressed. Thus, the work done by the capillary pressure for a certain time is given by

$$
\frac{\mathrm{d} W}{\mathrm{~d} t}=-p_{1} \frac{\mathrm{d} V_{1}}{\mathrm{~d} t}-p_{2} \frac{\mathrm{d} V_{2}}{\mathrm{~d} t}=-p_{1} \frac{\mathrm{d} V_{1}}{\mathrm{~d} t}+p_{2} \frac{\mathrm{d} V_{1}}{\mathrm{~d} t}=-p_{c} \frac{\mathrm{d} V_{1}}{\mathrm{~d} t}
$$

with the assumption $p_{c}$ is spatially constant along the interface. In this study, we use the the Young-Laplace equation

$$
p_{c}=\frac{2 \sigma \cos \theta}{r}
$$

to characterize the capillary pressure in the nanopore and the Weinaug-Katz correlation ${ }^{31}$

$$
\sigma=\left[\sum_{i=1}^{M}[\mathbf{P}]_{i}\left(\mathbf{n}_{i, 2}-\mathbf{n}_{i, 1}\right)\right]^{4}
$$

to model interfacial tension. 


\section{Evolution equations for moles and volume}

The evolution equations for the two-phase equilibria calculation are formulated on the basis of the first law of thermodynamics. The internal energy in unit time has following form

$$
\frac{\mathrm{d} U}{\mathrm{~d} t}=\frac{\mathrm{d} Q}{\mathrm{~d} t}+\frac{\mathrm{d} W}{\mathrm{~d} t}
$$

Substituting eq (3) and $U=F+T S_{\mathrm{sys}}$, into eq (6), we can derive

$$
\frac{\mathrm{d} S_{\text {sys }}}{\mathrm{d} t}=\frac{1}{T}\left(\frac{\mathrm{d} Q}{\mathrm{~d} t}\right)-\frac{1}{T}\left(\frac{\mathrm{d} F}{\mathrm{~d} t}\right)-\frac{p_{c}}{T}\left(\frac{\mathrm{d} V_{1}}{\mathrm{~d} t}\right)
$$

where $S_{\text {sys }}$ is the entropy of the system. Given that the total entropy has two components $S=S_{\mathrm{sys}}+S_{\text {env }}$, the entropy of the environment in the reverse process satisfies $\mathrm{d} S_{\text {env }}=-\mathrm{d} Q / T$ to keep the temperature constant. The time derivative of the total entropy is formulated as

$$
\frac{\mathrm{d} S}{\mathrm{~d} t}=\frac{\mathrm{d} S_{\text {sys }}}{\mathrm{d} t}+\frac{\mathrm{d} S_{\text {env }}}{\mathrm{d} t}=-\frac{1}{T}\left(\frac{\mathrm{d} F}{\mathrm{~d} t}\right)-\frac{p_{c}}{T}\left(\frac{\mathrm{d} V_{1}}{\mathrm{~d} t}\right)
$$

According to the mole and volume constraints, the Helmholtz free energy $F$ can be reduced into a function of $\mathbf{N}_{1}$ and $V_{1}$. Consequently, the partial derivatives of $F\left(\mathbf{N}_{1}, V_{1}\right)$ with respect to $N_{i, 1}$ and $V_{1}$ are $\partial F\left(\mathbf{N}_{1}, V_{1}\right) / \partial N_{i, 1}=\mu_{i, 1}-\mu_{i, 2}$ and $\partial F\left(\mathbf{N}_{1}, V_{1}\right) / \partial V_{1}=p_{2}-p_{1}$, where $\mu_{i, \alpha}=\mu_{i}\left(\mathbf{n}_{\alpha}\right)$ is the chemical potential of component $i$ in phase $\alpha$. By applying the chain rule, we can derive the time derivative of Helmholtz free energy as

$$
\frac{\mathrm{d} F\left(\mathbf{N}_{1}, V_{1}\right)}{\mathrm{d} t}=\sum_{i=1}^{M} \frac{\partial F}{\partial N_{i, 1}} \frac{\partial N_{i, 1}}{\partial t}+\frac{\partial F}{\partial V_{1}} \frac{\partial V_{1}}{\partial t}=\sum_{i=1}^{M}\left(\mu_{i}\left(\mathbf{n}_{1}\right)-\mu_{i}\left(\mathbf{n}_{2}\right)\right) \frac{\partial N_{i, 1}}{\partial t}+\left(p_{2}-p_{1}\right) \frac{\partial V_{1}}{\partial t}
$$

Substituting eq (9) into eq (8), the total entropy change becomes

$$
\frac{\mathrm{d} S}{\mathrm{~d} t}=\frac{1}{T} \sum_{i=1}^{M}\left(\mu_{i}\left(\mathbf{n}_{2}\right)-\mu_{i}\left(\mathbf{n}_{1}\right)\right) \frac{\partial N_{i, 1}}{\partial t}+\frac{1}{T}\left(p_{1}-p_{2}-p_{c}\right) \frac{\partial V_{1}}{\partial t}
$$


The Onsager's reciprocal principle ${ }^{32}$ indicates that there exists a symmetrical matrix $\boldsymbol{\Psi}=\left(\psi_{i, j}\right)_{i, j=1}^{M+1}$ such that

$$
\begin{aligned}
\frac{\partial N_{i, 1}}{\partial t} & =\sum_{j=1}^{M} \psi_{i, j}\left(\mu_{j}\left(\mathbf{n}_{2}\right)-\mu_{j}\left(\mathbf{n}_{1}\right)\right)+\psi_{i, M+1}\left(p_{1}-p_{2}-p_{c}\right) \\
\frac{\partial V_{1}}{\partial t} & =\sum_{j=1}^{M} \psi_{M+1, j}\left(\mu_{j}\left(\mathbf{n}_{2}\right)-\mu_{j}\left(\mathbf{n}_{1}\right)\right)+\psi_{M+1, M+1}\left(p_{1}-p_{2}-p_{c}\right)
\end{aligned}
$$

According to the second law of thermodynamics, the entropy $S$ never decreases, implying $\boldsymbol{\Psi}$ should be positive semi-definite. This can be proved by substituting eq (11) and eq (12) back into eq (10). Instead of constructing a specialized diagonal coefficient matrix ${ }^{30}$, a generalized coefficient matrix is computed using the conventional equilibrium conditions. It not only preserves entropy production with time, but allows to simultaneously solve the evolutionary equations, thereby improving the computational efficiency. Furthermore, it results into higher accuracy with the same or even less iterations. The computation of the generalized Onsager coefficient matrix will be discussed in the next section.

At the equilibrium state, the entropy reaches the maximum so that $\mathrm{d} S / \mathrm{d} t, \mathrm{~d} N_{i, 1} / \mathrm{d} t$ and $\mathrm{d} V_{1} / \mathrm{d} t$ all vanish. The chemical equilibrium $\left(\mu_{i}\left(\mathbf{n}_{2}\right)-\mu_{i}\left(\mathbf{n}_{1}\right)=0\right)$ and the pressure equilibrium $\left(p_{1}-p_{2}-p_{c}=0\right)$ are spontaneously satisfied. It is clear the confined equilibrium conditions will degenerate to the unconfined equilibrium conditions if capillary pressure is ignored.

\section{Thermodynamically-stable numerical algorithm}

In order to ensure the unconditional entropy production in discrete formulation, the convex splitting of Helmholtz free energy density and chemical potential plays an important role in constructing the thermodynamically stable numerical algorithm. This methodology has been extensively investigated in recent works. ${ }^{21,30,33-38}$ Also, we add an additional ideal term to construct a precise convex-concave splitting. As a result, the Helmholtz free energy 
density has the following form

$$
\begin{aligned}
f(\mathbf{n}) & =f^{\text {convex }}(\mathbf{n})+f^{\text {concave }}(\mathbf{n}) \\
f^{\text {convex }}(\mathbf{n}) & =(1+\lambda) f^{\text {ideal }}(\mathbf{n})+f^{\text {repulsion }}(\mathbf{n}) \\
f^{\text {concave }}(\mathbf{n}) & =f^{\text {attraction }}(\mathbf{n})-\lambda f^{\text {ideal }}(\mathbf{n})
\end{aligned}
$$

where $\lambda \in(0.1,10)$ for practical computation. Accordingly, the chemical potential $\mu_{i}(\mathbf{n})$ has two components $\mu_{i}^{\text {convex }}(\mathbf{n})$ and $\mu_{i}^{\text {concave }}(\mathbf{n})$, where

$$
\begin{gathered}
\mu_{i}^{\text {convex }}(\mathbf{n})=\frac{\partial f^{\text {convex }}}{\partial n_{i}}=(1+\lambda) \frac{\partial f^{\text {ideal }}}{\partial n_{i}}+\frac{\partial f^{\text {repulsion }}}{\partial n_{i}} \\
\mu_{i}^{\text {concave }}(\mathbf{n})=\frac{\partial f^{\text {concave }}}{\partial n_{i}}=\frac{\partial f^{\text {attraction }}}{\partial n_{i}}-\lambda \frac{\partial f^{\text {ideal }}}{\partial n_{i}}
\end{gathered}
$$

To construct an unconditionally stable numerical algorithm, the semi-implicit timemarching scheme is applied to discretize eq (11) and eq (12) by treating the convex part of chemical potential implicitly and the concave part of chemical potential explicitly. A generalized Onsager coefficient matrix is generated, which allows the evolution equations to be solved simultaneously. The temporal discretization of mole evolutionary equation and volume evolutionary equation are given by

$$
\begin{aligned}
& \frac{N_{i, 1}^{k+1}-N_{i, 1}^{k}}{\delta t}=\sum_{j=1}^{M} \psi_{i, j}\left(\mu_{j, 2}^{k+1}-\mu_{j, 1}^{k+1}\right)+\psi_{i, M+1}\left(p_{1}^{k+1}-p_{2}^{k+1}-p_{c}^{k+1}\right) \\
& \frac{V_{1}^{k+1}-V_{1}^{k}}{\delta t}=\sum_{j=1}^{M} \psi_{M+1, j}\left(\mu_{j, 2}^{k+1}-\mu_{j, 1}^{k+1}\right)+\psi_{M+1, M+1}\left(p_{1}^{k+1}-p_{2}^{k+1}-p_{c}^{k+1}\right)
\end{aligned}
$$


where

$$
\begin{aligned}
& \mu_{j, 1}^{k+1}=\mu_{j}^{\text {convex }}\left(\mathbf{n}_{1}^{k+1}\right)+\mu_{j}^{\text {concave }}\left(\mathbf{n}_{1}^{k}\right) \\
& \mu_{j, 2}^{k+1}=\mu_{j}^{\text {convex }}\left(\mathbf{n}_{2}^{k+1}\right)+\mu_{j}^{\text {concave }}\left(\mathbf{n}_{2}^{k}\right) \\
& p_{1}^{k+1}=\sum_{i=1}^{M}\left(n_{i, 1}^{k+1} \mu_{i}^{\text {convex }}\left(\mathbf{n}_{1}^{k+1}\right)+n_{i, 1}^{k} \mu_{i}^{\text {concave }}\left(\mathbf{n}_{1}^{k}\right)\right)-f^{\text {convex }}\left(\mathbf{n}_{1}^{k+1}\right)-f^{\text {concave }}\left(\mathbf{n}_{1}^{k}\right) \\
& p_{2}^{k+1}=\sum_{i=1}^{M}\left(n_{i, 2}^{k+1} \mu_{i}^{\text {convex }}\left(\mathbf{n}_{2}^{k+1}\right)+n_{i, 2}^{k} \mu_{i}^{\text {concave }}\left(\mathbf{n}_{2}^{k}\right)\right)-f^{\text {convex }}\left(\mathbf{n}_{2}^{k+1}\right)-f^{\text {concave }}\left(\mathbf{n}_{2}^{k}\right) \\
& p_{c}^{k+1}=p_{c}\left(\mathbf{N}_{1}^{k+1}, V_{1}^{k+1}\right)
\end{aligned}
$$

It can be demonstrated the semi-implicit time-marching scheme is thermodynamically stable. The detailed proof is included in the Supporting Information.

As discussed before, the Onsager coefficient matrix $\boldsymbol{\Psi}$ should be positive semi-definite in order to satisfy the principle of entropy production. Kou and Sun ${ }^{30}$ constructed a diagonal Onsager matrix using the diffusion coefficient of each component and other two nonzero parameters. Using such a specialized coefficient matrix in which all diagonal elements are positive, they solved their proposed model sequentially. In this study, we propose a generalized Onsager coefficient matrix based upon the equilibrium conditions of unconfined VT-flash problems, which has the following form

$$
\boldsymbol{\Psi}=\left[\begin{array}{cc}
A & B \\
B^{T} & C
\end{array}\right]
$$

where $A=\partial\left(\mu_{i, 2}-\mu_{i, 1}\right) / \partial N_{i, 1}, B=\partial\left(\mu_{i, 2}-\mu_{i, 1}\right) / \partial V_{1}=\partial\left(p_{1}-p_{2}\right) / \partial N_{i, 1}$, and $C=$ $\partial\left(p_{1}-p_{2}\right) / \partial V_{1}$. If $\boldsymbol{\Psi}$ is sufficiently positive definite, no additional operations are required. Otherwise, the revised modified Cholesky factorization suggested by Smejkal and Mikyška ${ }^{23}$ is applied to ensure its positive definiteness. A diagonal matrix $\boldsymbol{E}$ with suitable non-negative entries will be added so that $\boldsymbol{\Psi}+\boldsymbol{E}$ becomes sufficiently positive definite. It is clear $\boldsymbol{E}$ is a zero matrix if $\boldsymbol{\Psi}$ is safely positive definite. In addition, the infinity norm of $\boldsymbol{E}$ should not be 
much larger than the most negative eigenvalue of $\boldsymbol{\Psi} .{ }^{39}$ The modified coefficient matrix results in fast convergence of the Newton method to the local maximum, with entropy increasing in each iteration. To obtain a better conditioned problem, a right preconditioner is designed in terms of the structure of the Jacobian matrix, leading to the better performance of the Newton-Raphson method.

\section{Numerical simulation}

The numerical simulation is configured in this section. In this study, we use $\lambda=0.1$. The mean pore radius $r=10 \mathrm{~nm}$ and the contact angle $\theta=\pi / 6$. We assume the system has unit volume $\left(V=1 \mathrm{~m}^{3}\right)$. In order to provide better initial approximations for the equilibrium problem with capillarity, the capillary effect is also taken into account in VTstability testing. Here we apply the confined stability analysis proposed by Kou and Sun ${ }^{30}$ to initialize phase equilibrium calculation. The Newton-Raphson method with line search is used to solve nonlinear systems and the number of Newton iteration is 10 . We terminate Newton iterations if the Euclidean norm of the objective functions is less than $10^{-5}$. In stability testing, the total number of time steps is 200 and the stopping criterion is either $\left\|\mathbf{n}^{k}-\mathbf{n}^{k-1}\right\|_{2}<10^{-5}$ or $\left|\left(D^{k}-D^{k-1}\right) / D^{k-1}\right|<10^{-8}$, where $D$ is the value of TPD function. Furthermore, the total number of time steps for phase equilibrium calculation is 1000 and the terminating criterion is defined as $\max \left(\left\|\Delta \mu^{k}\right\|,\left|\Delta p^{k}\right|\right)<10^{-6}$ with

$$
\begin{aligned}
\left\|\Delta \mu^{k}\right\| & =\frac{\left\|\mu\left(\mathbf{n}_{1}^{k}\right)-\mu\left(\mathbf{n}_{2}^{k}\right)\right\|_{2}}{\max \left(\left\|\mu\left(\mathbf{n}_{1}^{k}\right)\right\|_{2},\left\|\mu\left(\mathbf{n}_{2}^{k}\right)\right\|_{2}\right)} \\
\left|\Delta p^{k}\right| & =\frac{\left|p_{1}^{k}-p_{2}^{k}-p_{c}^{k}\right|}{\max \left(\left|p_{1}^{k}\right|,\left|p_{2}^{k}\right|\right)}
\end{aligned}
$$

It is worth noting that adaptive time step is applied in both stability analysis and phase equilibrium calculation based on the difference of the entropy increment at two neighboring time steps. Table 1 and Table 2 present parameters of the Peng-Robinson EOS and parachors for 
all studied components. Binary interaction coefficients for the binary- and multi-component mixtures can be found in ref. 25 .

Table 1: Parameters of the Peng-Robinson EOS for all components in numerical examples.

\begin{tabular}{lllll}
\hline Component & $T_{\mathrm{c}, i}(\mathrm{~K})$ & $P_{\mathrm{c}, i}(\mathrm{MPa})$ & $M_{\mathrm{w}, i}(\mathrm{~g} / \mathrm{mol})$ & $\omega_{i}$ \\
\hline $\mathrm{N}_{2}$ & 126.21 & 3.390 & 28.01 & 0.0390 \\
$\mathrm{CO}_{2}$ & 304.14 & 7.375 & 44.01 & 0.2390 \\
$\mathrm{C}_{1}$ & 190.56 & 4.599 & 16.04 & 0.0110 \\
$\mathrm{C}_{3}$ & 369.83 & 4.248 & 44.10 & 0.1530 \\
$\mathrm{nC}_{4}$ & 424.90 & 3.800 & 58.12 & 0.1930 \\
$\mathrm{nC}_{5}$ & 469.70 & 3.370 & 72.15 & 0.2510 \\
$\mathrm{C}_{6}$ & 507.40 & 3.012 & 86.20 & 0.2960 \\
$\mathrm{nC}_{10}$ & 617.70 & 2.110 & 142.28 & 0.4890 \\
$\mathrm{PC}_{1}$ & 333.91 & 5.329 & 34.64 & 0.1113 \\
$\mathrm{PC}_{2}$ & 456.25 & 3.445 & 69.52 & 0.2344 \\
$\mathrm{PC}_{3}$ & 590.76 & 2.376 & 124.57 & 0.4470 \\
$\mathrm{C}_{12+}$ & 742.58 & 1.341 & 248.30 & 0.9125 \\
\hline
\end{tabular}

Table 2: Parachor values of all components in numerical examples.

\begin{tabular}{lllllllllllll}
\hline Component & $\mathrm{N}_{2}$ & $\mathrm{CO}_{2}$ & $\mathrm{C}_{1}$ & $\mathrm{C}_{3}$ & $\mathrm{nC}_{4}$ & $\mathrm{nC}_{5}$ & $\mathrm{C}_{6}$ & $\mathrm{nC}_{10}$ & $\mathrm{PC}_{1}$ & $\mathrm{PC}_{2}$ & $\mathrm{PC}_{3}$ & $\mathrm{C}_{12+}$ \\
\hline$[\mathbf{P}]_{i}$ & $41.0^{\mathrm{a}}$ & 78.0 & 77.3 & 150.3 & 189.9 & 233.9 & 271.0 & 433.5 & $97.8^{\mathrm{b}}$ & $202.5^{\mathrm{b}}$ & $356.8^{\mathrm{b}}$ & $655.0^{\mathrm{b}}$ \\
\hline
\end{tabular}

a The parachor of nitrogen in hydrocarbon mixtures.

b Parachors of crude oil fractions are calculated from eq (3) in ref. 40.

\section{Results and discussion}

In this section, the thermodynamically stable phase equilibrium calculation in the presence of capillary pressure is tested for single-, binary-, and multi-component mixtures that have at most two phases at the given temperature and overall molar concentration. We partition the computational domain into a $50 \times 50$ grid to investigate phase compositions and distributions. To demonstrate the broad applicability of the proposed method, we compute the unconfined VT-flash problems and compare our results with the published ones. ${ }^{19,25}$ Considering phase properties (e.g. volume and density) gradually change over the two-phase region, the outcome of stability testing that is close to the equilibrium condition of neigh- 
boring states is used to initialize phase equilibrium calculation, thereby leading to a phase transition process with uniform phase identification.

\section{Example 1}

In the first example, the phase equilibrium of pure n-butane $\left(\mathrm{nC}_{4}\right)$ is investigated. The overall molar density ranges from 0 to $12000 \mathrm{~mol}$ and the temperature varies from 300 to 430 K. Figure 1 displays the global minimum of TPD function with and without capillary effect, respectively. The solid black curve represents the approximate boundary between the single-phase region and the two-phase region. It can be seen the global minimum of TPD function has some difference under capillary pressure, but the change of the phase boundary is indistinguishable. Thus, a P-T phase diagram is created in order to clearly reveal the effect of capillary pressure on phase equilibria, as shown in Figure 2.
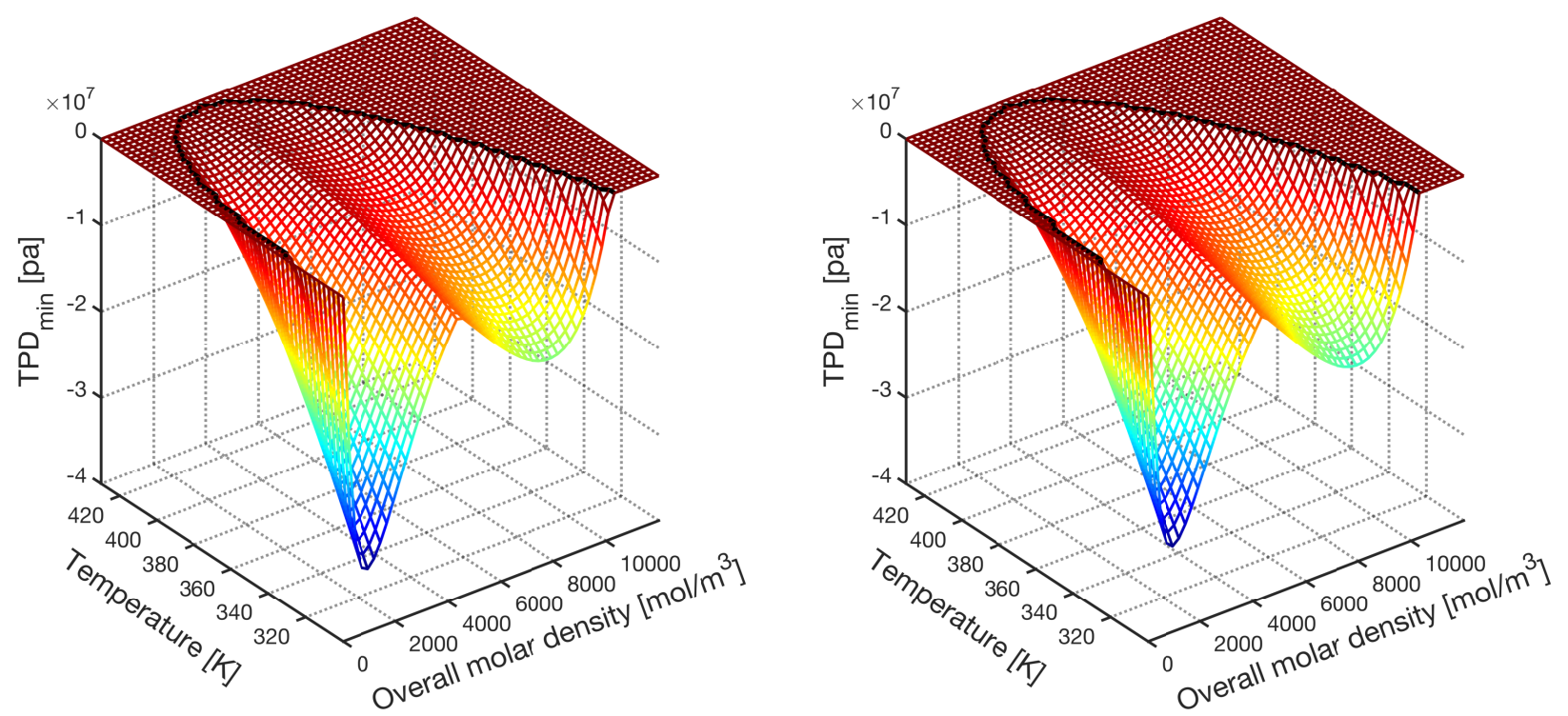

Figure 1: Global minimum of TPD function for pure $\mathrm{nC}_{4}$ in the presence of capillary pressure (left) and in the absence of capillary pressure (right), respectively. 


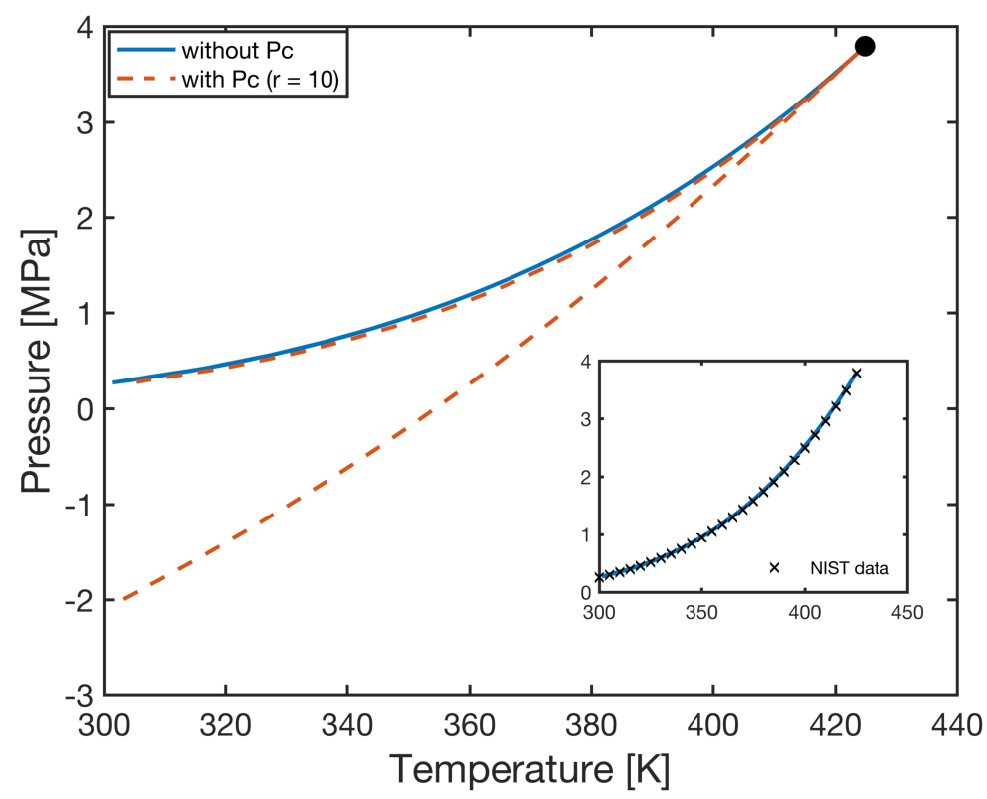

Figure 2: Phase diagram for pure $\mathrm{nC}_{4}$ with and without capillary pressure. The black point represents the approximate critical point. The inset figure shows the computed saturation pressure and the data obtained from NIST chemistry webbook.

To construct the phase diagram, the pressures of both phases along the phase boundary are computed. Here a finer mesh is used to achieve the smooth bubble point and dew point curves. When capillary pressure is ignored, the computed vapor pressure and liquid pressure are almost same, as the result of the unconfined pressure equilibrium condition. We calculate the average pressure of vapor and liquid phases and compare our results with the data collected from the NIST chemistry webbook. It can be seen in the inset figure that our computational results are highly consistent with the published data. As a result of the capillary effect, the bubble point pressure differs from the dew point pressure. The upper dashed line represents the dew point curve, and the lower dashed line represents the bubble point curve. It is clear the bubble point pressure deviates from its bulk value more significantly than the dew point pressure. The significant decrease of bubble point pressure results in remarkable expansion of the single liquid region, while the insignificant variation of dewpoint curve unremarkably changes the phase boundary between the single vapor region and vapor-liquid region. We also observe that the approximate critical point does not change by introducing capillary pressure, shown as the black point in Figure 2. 
The computed critical pressure and temperature are $3.7944 \mathrm{MPa}$ and $424.800 \mathrm{~K}$, which are very close to the critical pressure $3.7960 \mathrm{MPa}$ and $425.125 \mathrm{~K}$ given by the NIST chemistry webbook.
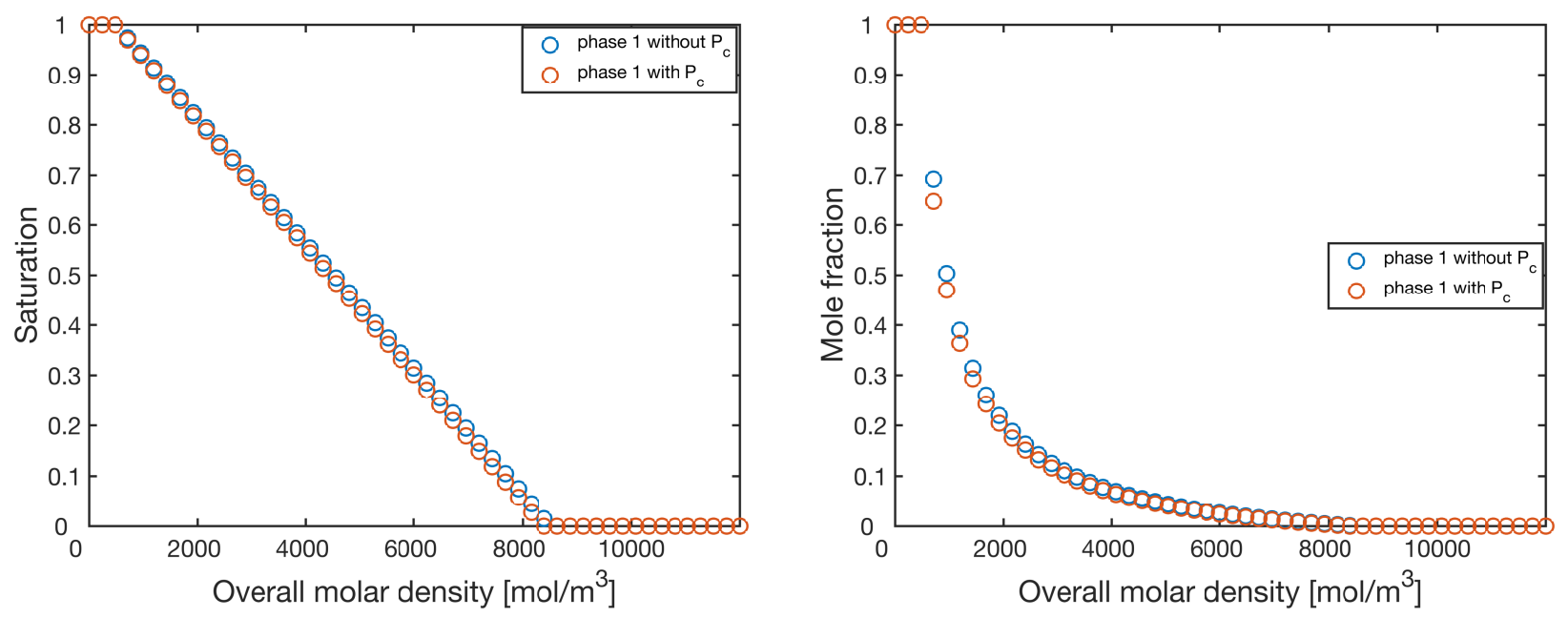

Figure 3: Saturation (left) and mole fraction (right) of phase 1 as a function of the overall molar density (left) at $T=360$ with and without capillary pressure.

Figure 3 shows the saturation and mole fraction of phase 1 as a function of the overall molar density with and without capillary pressure at the specified temperature. Our simulation results match up with the published results ${ }^{30}$ very well. Both saturation and mole fraction are compressed in the presence of the capillary effect. As the overall molar density increases, the volume contraction becomes larger in the two-phase region and the mole fraction reduction is weakened.

\section{Example 2}

The binary mixture of methane $\left(\mathrm{C}_{1}\right)$ and n-pentane $\left(\mathrm{nC}_{5}\right)$ is considered in this example. The overall mole fraction of $\mathrm{C}_{1}$ and $\mathrm{nC}_{5}$ is 0.547413 and 0.452587 , respectively. Figure 4 displays the global minimum of TPD function with the overall molar density $c \in[0,18000] \mathrm{mol} / \mathrm{m}^{3}$ and temperature $T \in[320,430] \mathrm{K}$. There is only one two-phase region delineated by the solid black curve. In the absence of capillary pressure, our simulation results agree with the results of example 2 in ref. 19. 

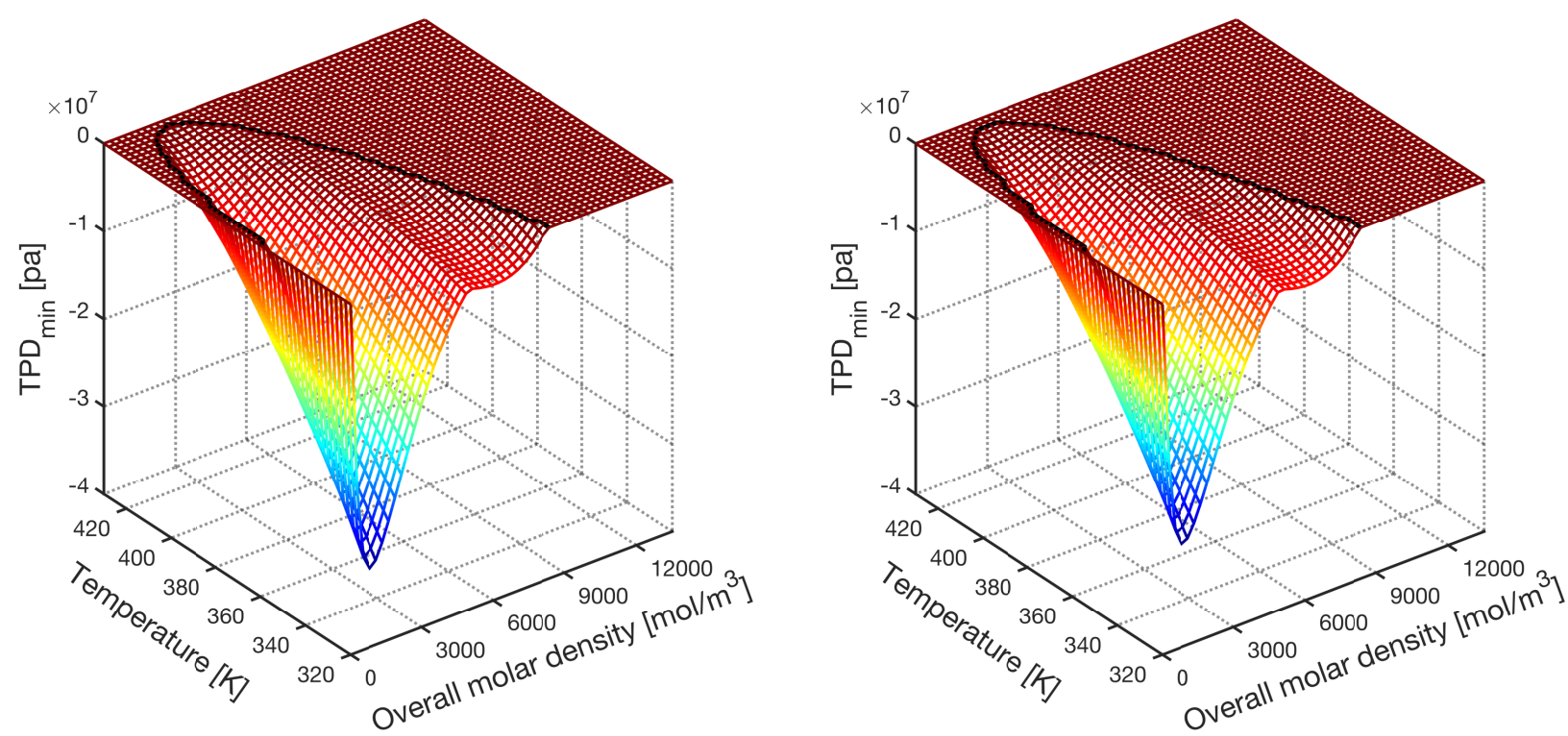

Figure 4: Global minimum of TPD function for the binary mixture of $\mathrm{C}_{1}$ and $\mathrm{nC}_{5}$ in the presence of capillary pressure (left) and in the absence of capillary pressure (right), respectively.

Capillary pressure reshapes the bulk phase envelope, as shown in Figure 5. The bubble point cruve is suppressed, which explains why the boundary between the single-liquid region and vapor-liquid region contracts at large overall concentration in Figure 4 (left). On the other hand, the dew point curve slightly expands outward, accounting for the insignificant expansion of the phase boundary at small overall concentration. It is worth mentioning that the expanded dew point curve is attributed to the complex dew point behavior. A similar observation is made in some references ${ }^{10,12,14}$ that the variation of dew point pressure is location-dependent in the presence of capillary pressure: dew point pressure increases in the upper branch of dewpoint curve and decreases in the lower branch. The overall effect of different dew point pressure changes expands the vapor-liquid region, which is consistent to our simulation results. When the pore radius decreases to $5 \mathrm{~nm}$, the phase envelope deviates more significantly, which results from the amplified capillary effect. It should be mentioned the approximate critical point is slightly different when the pore radius changes from $10 \mathrm{~nm}$ to $5 \mathrm{~nm}$. Actually, it is indirectly determined by locating the smallest pressure difference along the phase boundary, given the fact that phase properties become similar near the 
critical point so that capiilary pressure will vanish. The black point in Figure 5 represents the approximate critical point, which is computed by averaging the critical pressures and temperatures at different pore radius. As the condition of the hydrocarbon mixture goes far away from the critical point, the bubble point suppression becomes larger.

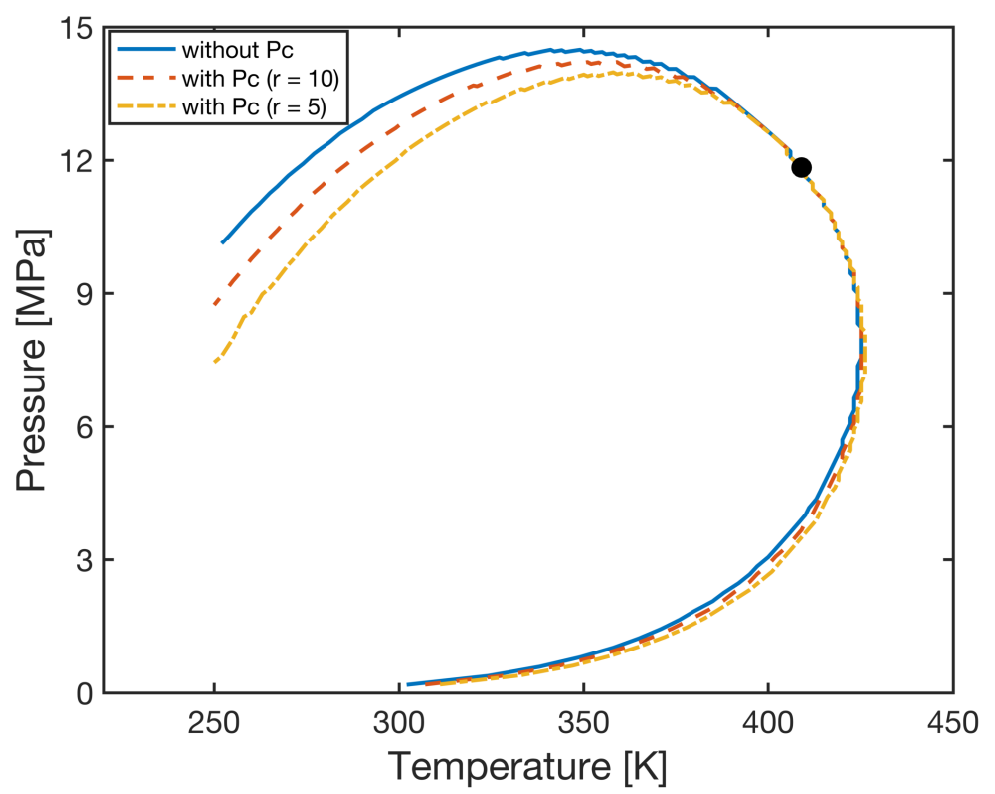

Figure 5: Phase envelope for the binary mixture of $\mathrm{C}_{1}$ and $\mathrm{nC}_{5}$ with and without capillary pressure. The black point represents the approximate critical point.

Figure 6 presents the saturation and mole fraction of phase 1 as a function of the overall molar density with and without capillary pressure. Figure 7 compares the mole fractions of $\mathrm{C}_{1}$ and $\mathrm{nC}_{5}$ in the two-phase region. It is interesting that the mole fractions of $\mathrm{C}_{1}$ increase in both phases under the capillary effect, which seems to be contradictory to the mole constraint. However, considering the mole number of phase 1 becomes smaller in the presence of capillary pressure, we cannot conclude the capillary confinement increases the mole numebr of $\mathrm{C}_{1}$ in phase 1 . As a result, the mole fraction of $\mathrm{C}_{1}$ in phase 2 could increase, since the reduced mole fraction of phase 1 balances out the mole fraction increase of $\mathrm{C}_{1}$ in phase 1. 

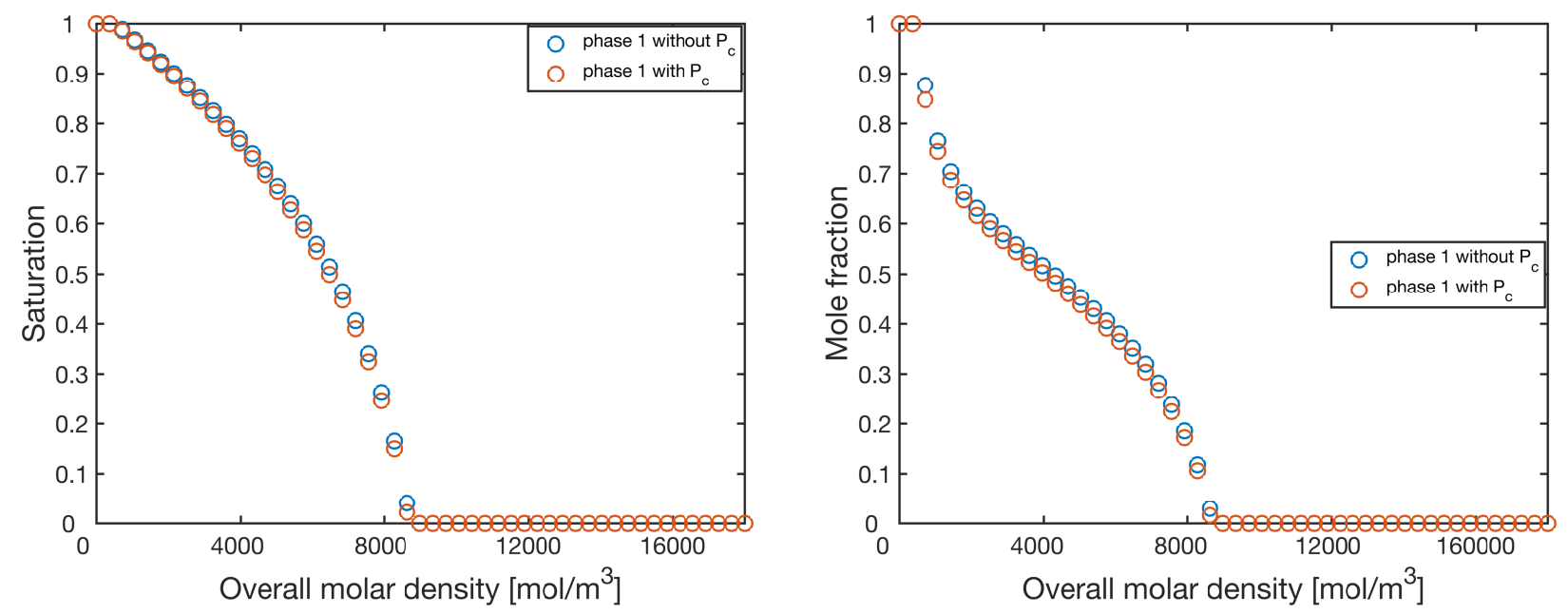

Figure 6: Saturation (left) and mole fraction (right) of phase 1 as a function of the overall molar density at $T=371 \mathrm{~K}$ with and without capillary pressure.
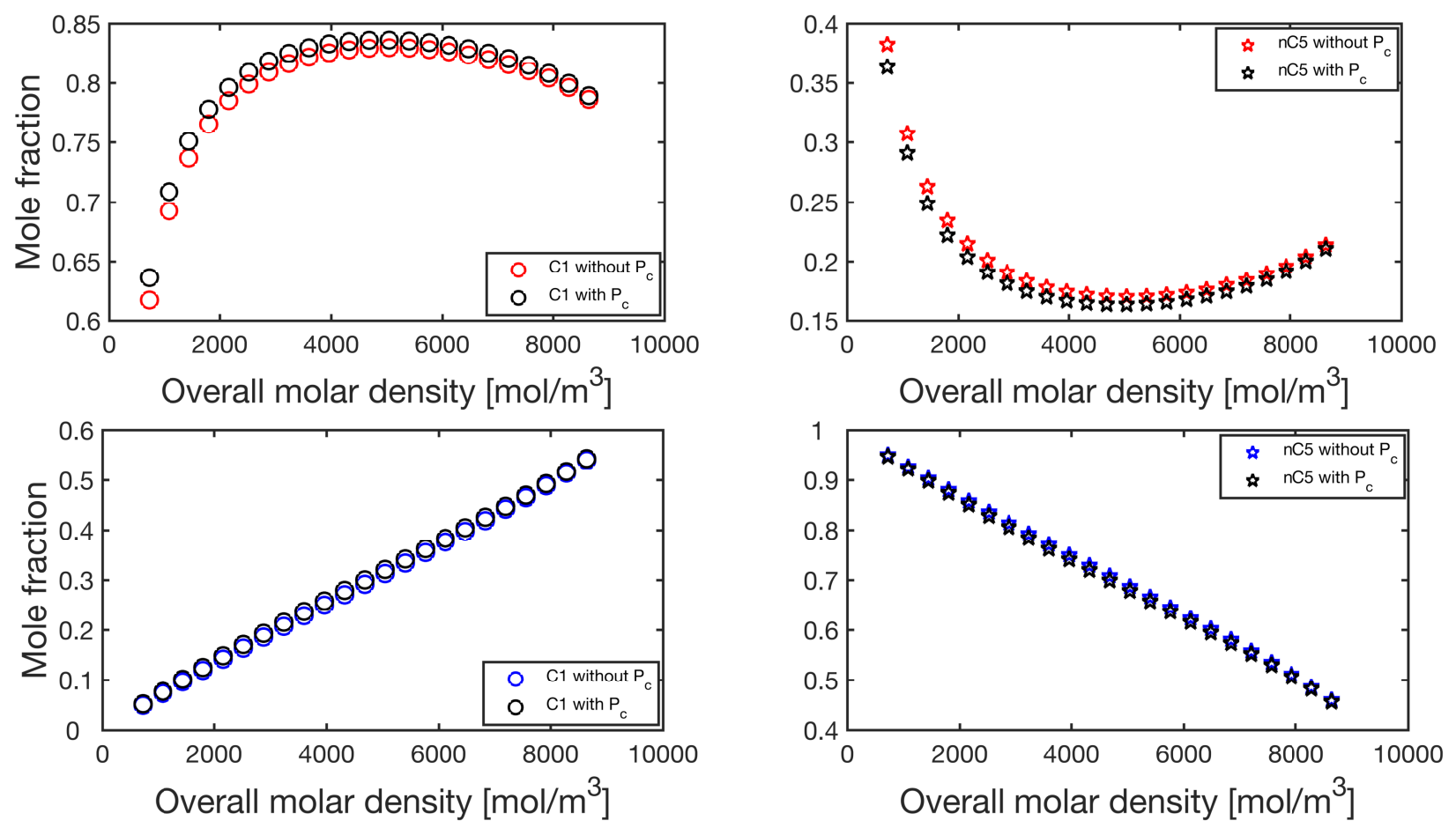

Figure 7: Mole fractions of $\mathrm{C}_{1}$ and $\mathrm{nC}_{5}$ in the two-phase region of phase 1 (top) and phase 2 (bottom) as a function of the overall molar density at $T=371 \mathrm{~K}$ with and without capillary pressure. 


\section{Example 3}

In the third example, we investigate the two-phase equilibrium for the four-component mixture of nitrogen $\left(\mathrm{N}_{2}\right)$, methane $\left(\mathrm{C}_{1}\right)$, propane $\left(\mathrm{C}_{3}\right)$ and n-decane $\left(\mathrm{nC}_{10}\right)$, with mole fractions $z_{\mathrm{N}_{2}}=0.2463, z_{\mathrm{C}_{1}}=0.2208, z_{\mathrm{C}_{3}}=0.2208$ and $z_{\mathrm{nC}_{10}}=0.3121$. Figure 8 exhibits the global minimum of TPD function at the overall molar density $c \in[0,12000] \mathrm{mol} / \mathrm{m}^{3}$ and temperature $T \in[250,650] \mathrm{K}$. Similar to results for the last example, a unique two-phase region is detected either including or excluding capillary pressure. When capillary effect is negligible, our simulation results are almost same to the results of example 5 in ref. 19 at the specified temperature.
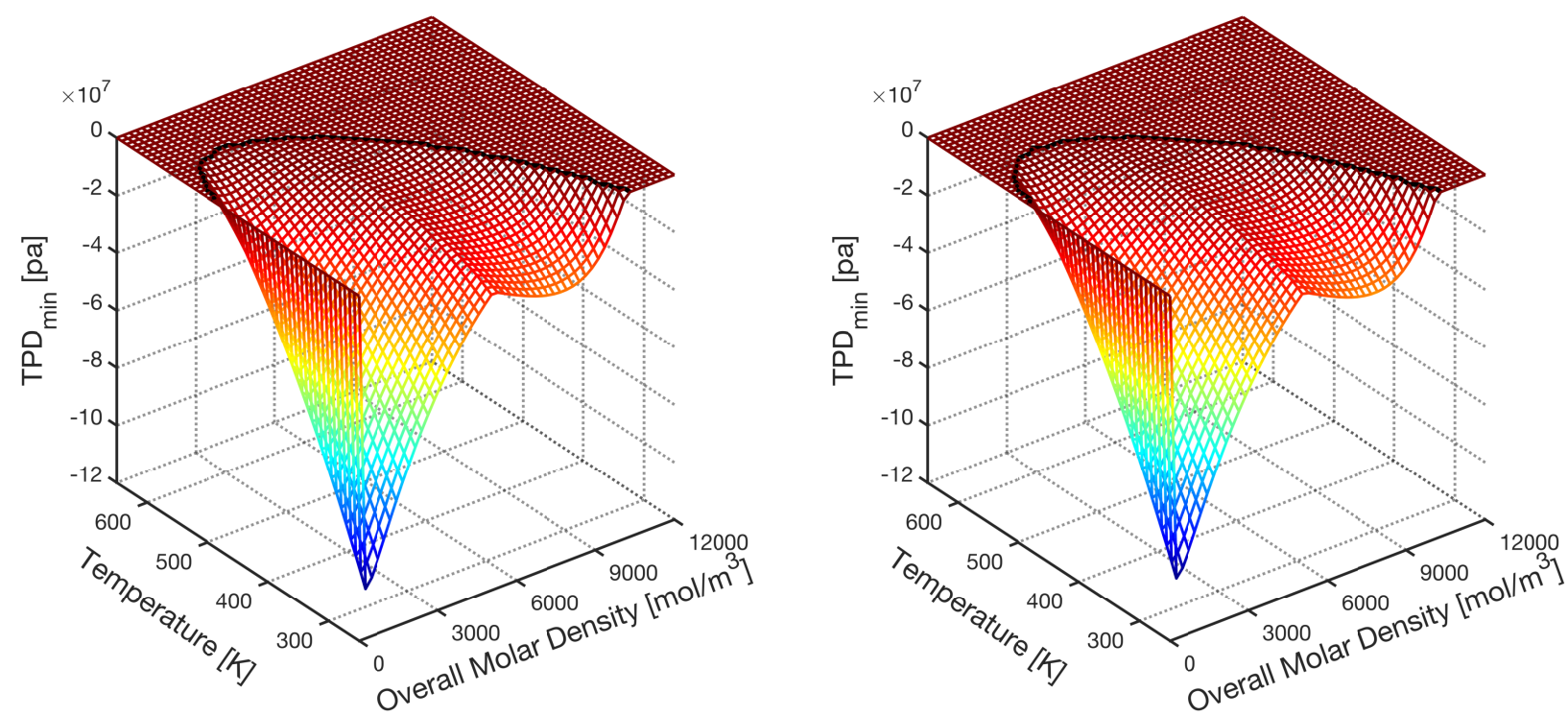

Figure 8: Global minimum of TPD function for the four-component mixture of $\mathrm{N}_{2}, \mathrm{C}_{1}, \mathrm{C}_{3}$ and $\mathrm{nC}_{10}$ in the presence of capillary pressure (left) and in the absence of capillary pressure (right), respectively.

As a result of capillary equilibrium, the boundary between the single-liquid region and vapor-liquid region in Figure 8 (left) is observed to shrink at high overall molar density, meaning the bubble point pressure is suppressed as shown in Figure 9. At low overall molar density, the phase boundary slightly expands, resulting from insignificant expansion of dew point curve toward the single-vapor region. The suppression of bubble point pressure becomes larger when the condition is further away from the approximate critical point. 
Figure 10 displays saturation and mole fraction profiles of phase 1 as a function of the overall concentration at the given temperature. It can be seen the capillary compression is unremarkable as the molar concentration increases. Mole fractions of all four components in the two-phase region of phase 1 and phase 2 with and without capillary effect are compared in Figure 11 and Figure 12.

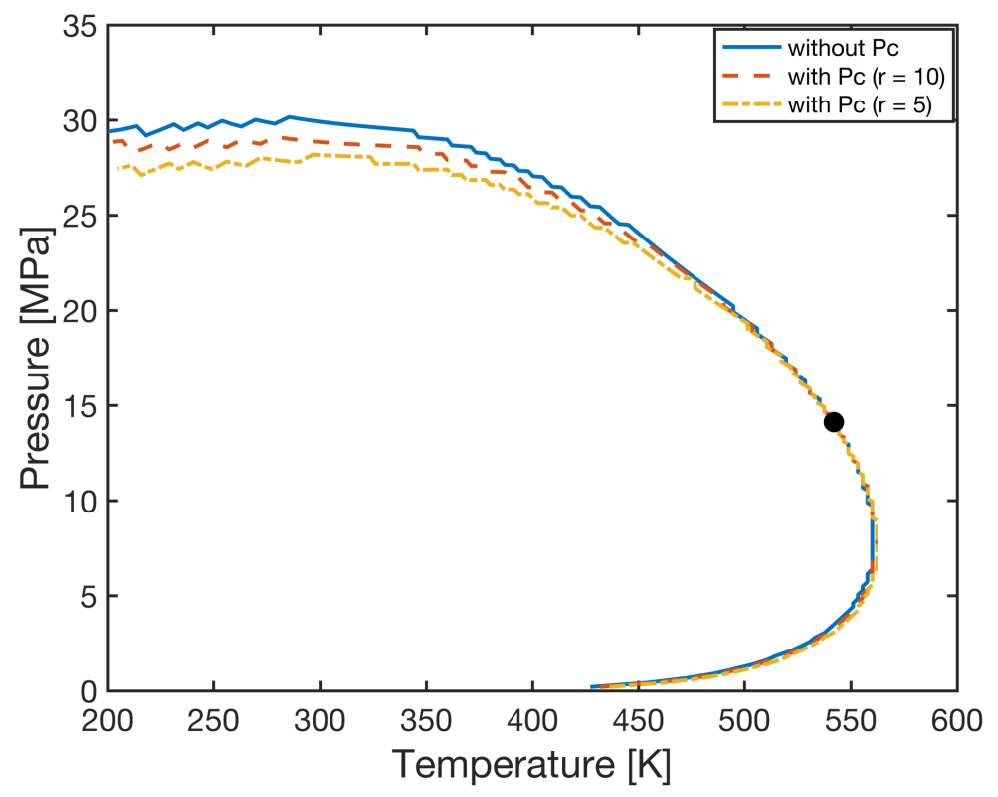

Figure 9: Phase envelope for the four-component mixture of $\mathrm{N}_{2}, \mathrm{C}_{1}, \mathrm{C}_{3}$ and $\mathrm{nC}_{10}$ with and without capillary pressure. The black point represents the approximate critical point.
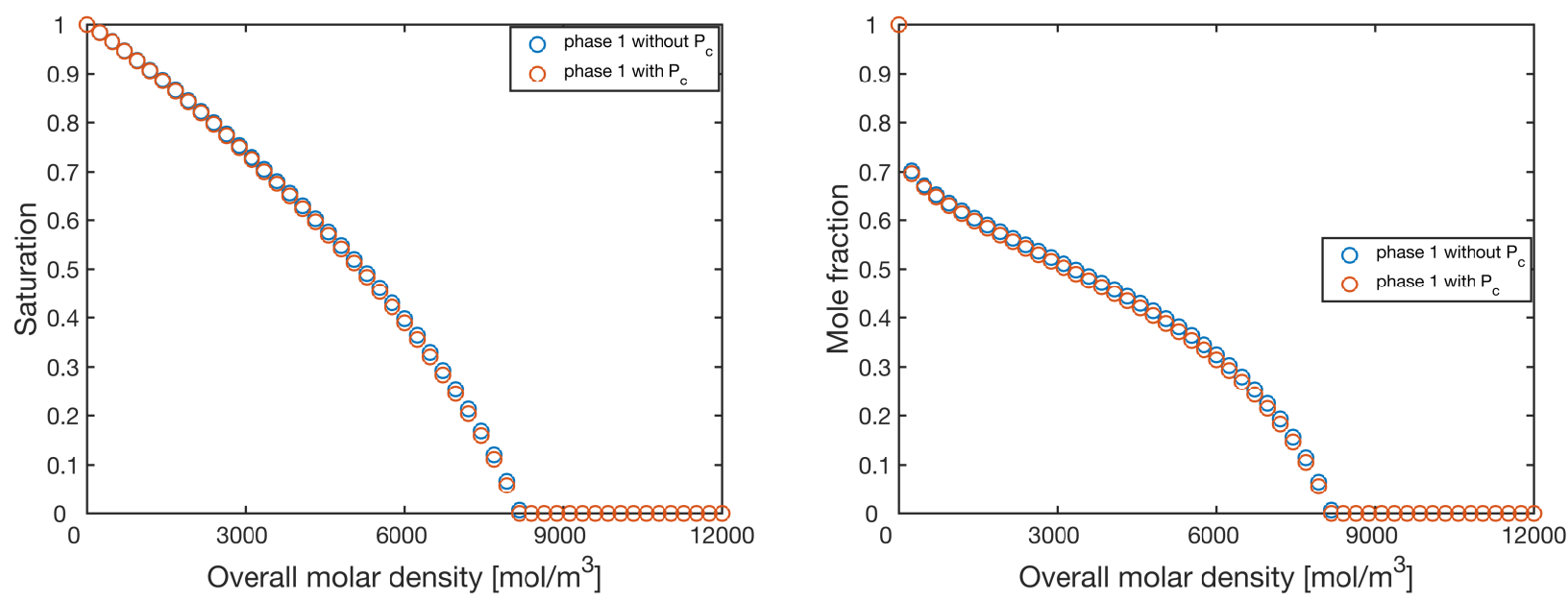

Figure 10: Saturation (left) and mole fraction (right) of phase 1 as a function of the overall molar density at $T=394 \mathrm{~K}$ with and without capillary pressure. 

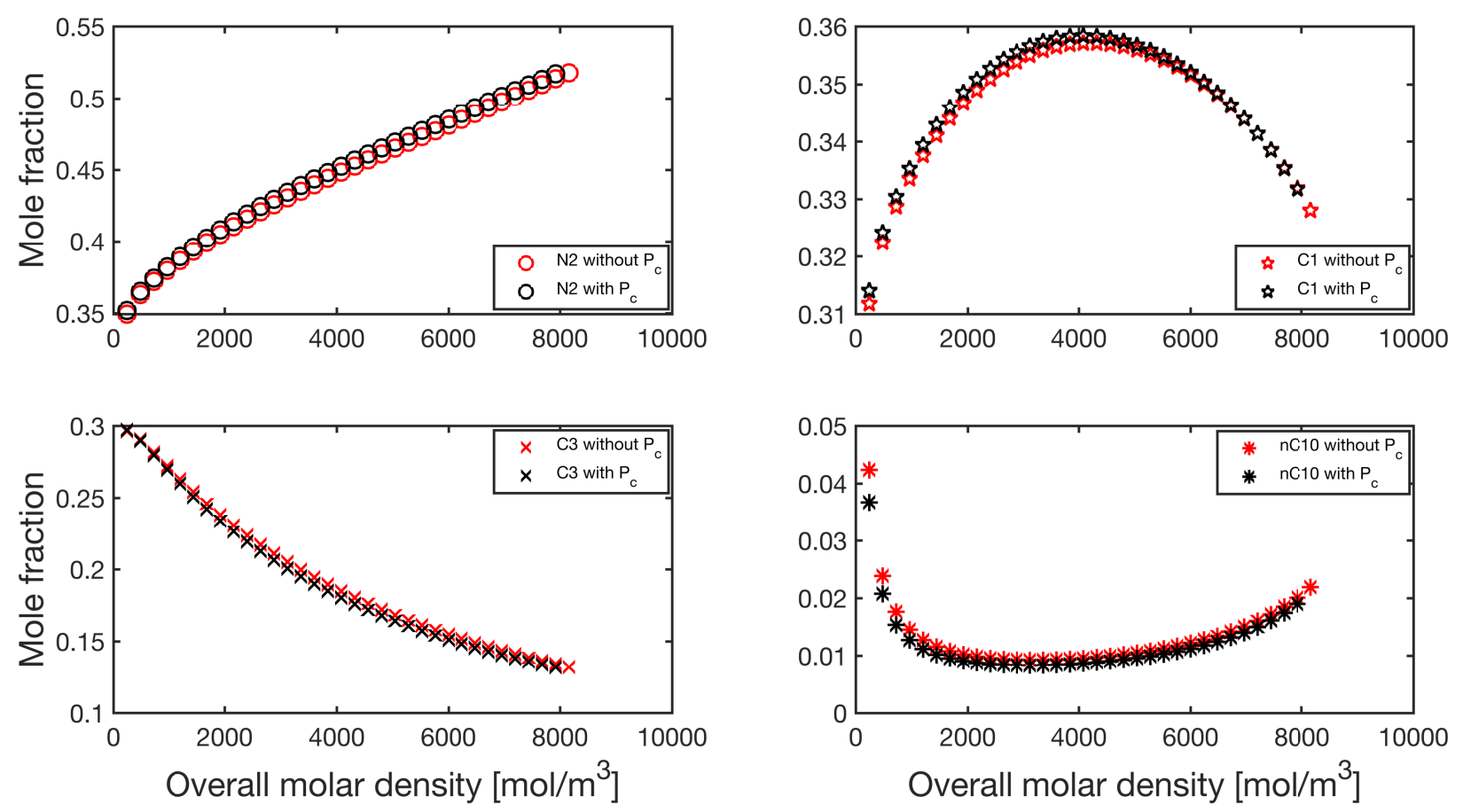

Figure 11: Mole fractions of all four components in the two-phase region of phase 1 as a function of the overall molar density at $T=394 \mathrm{~K}$ with and without capillary pressure.
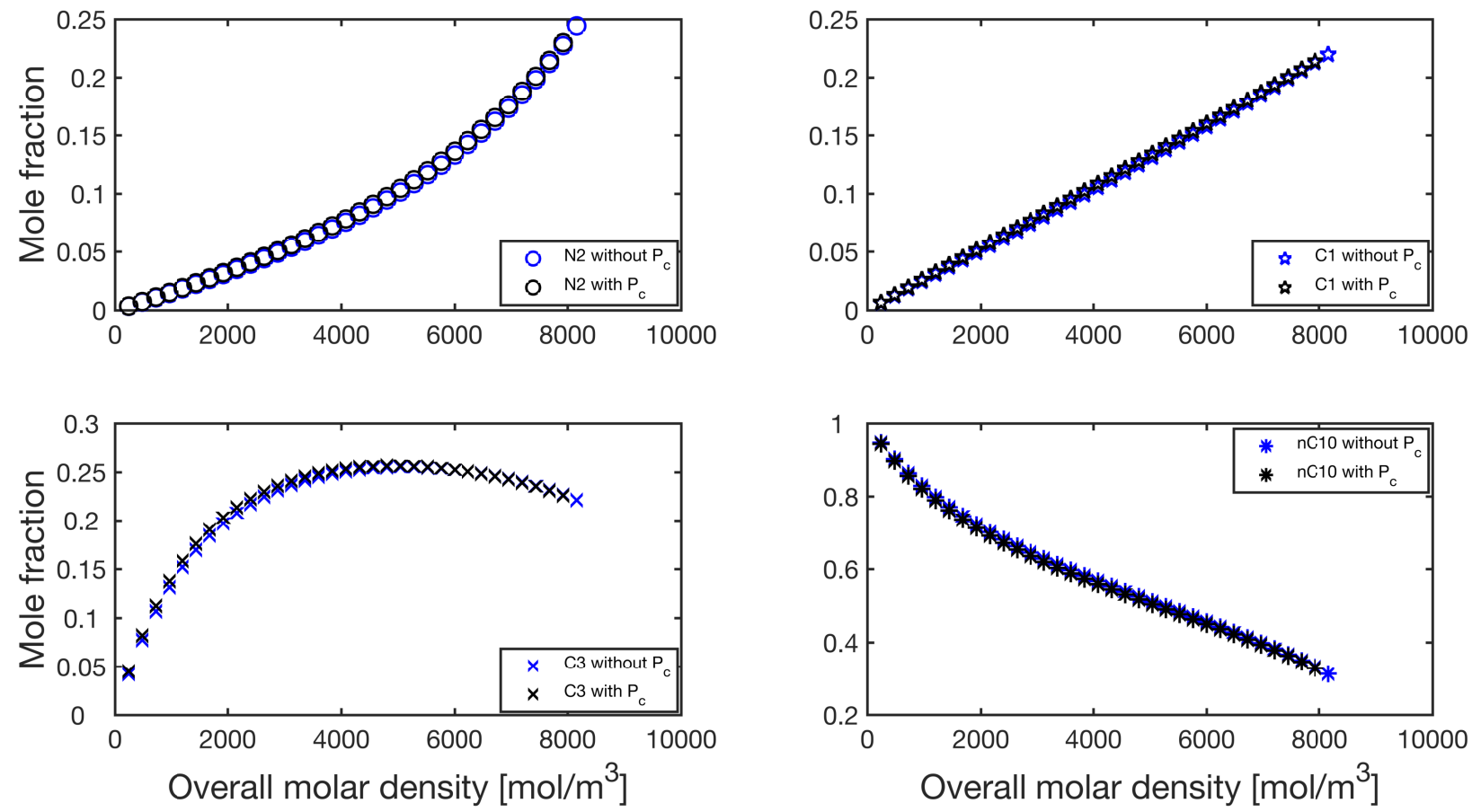

Figure 12: Mole fractions of all four components in the two-phase region of phase 2 as a function of the overall molar density at $T=394 \mathrm{~K}$ with and without capillary pressure. 


\section{Example 4}

The last example considers a seven-component mixture consisting of nitrogen $\left(\mathrm{N}_{2}\right)$, carbon dioxide $\left(\mathrm{CO}_{2}\right)$, methane $\left(\mathrm{C}_{1}\right)$ and four hydrocarbon pseudo-components $\mathrm{PC}_{1}, \mathrm{PC}_{2}$, $\mathrm{PC}_{3}$ and $\mathrm{C}_{12+}$, with mole fractions $z_{\mathrm{N}_{2}}=0.000131, z_{\mathrm{CO}_{2}}=0.568185, z_{\mathrm{C}_{1}}=0.246739$, $z_{\mathrm{PC}_{1}}=0.086275, z_{\mathrm{PC}_{2}}=0.033722, z_{\mathrm{PC}_{3}}=0.037006$ and $z_{\mathrm{C}_{12+}}=0.027941$. Phase equilibrium calculation for this complex fluid mixture is performed at the overall molar density $c \in[0,22500] \mathrm{mol} / \mathrm{m}^{3}$ and temperature $T \in[250,650] \mathrm{K}$. An additional unstable region is observed in the bottom-right corner of Figure 13, implying another two-phase region exists at very high molar concentration. Figure 14 shows the mass density profile as a function of the overall molar density without capillary effect. The first two-phase region consists of both vapor and liquid phases, and the second one could be the liquid-liquid region due to the large densities of both phases. It is noted that the mass density of phase 1 is so large that phase 1 may not be the vapor phase right before the fluid mixture enters the single-phase region.
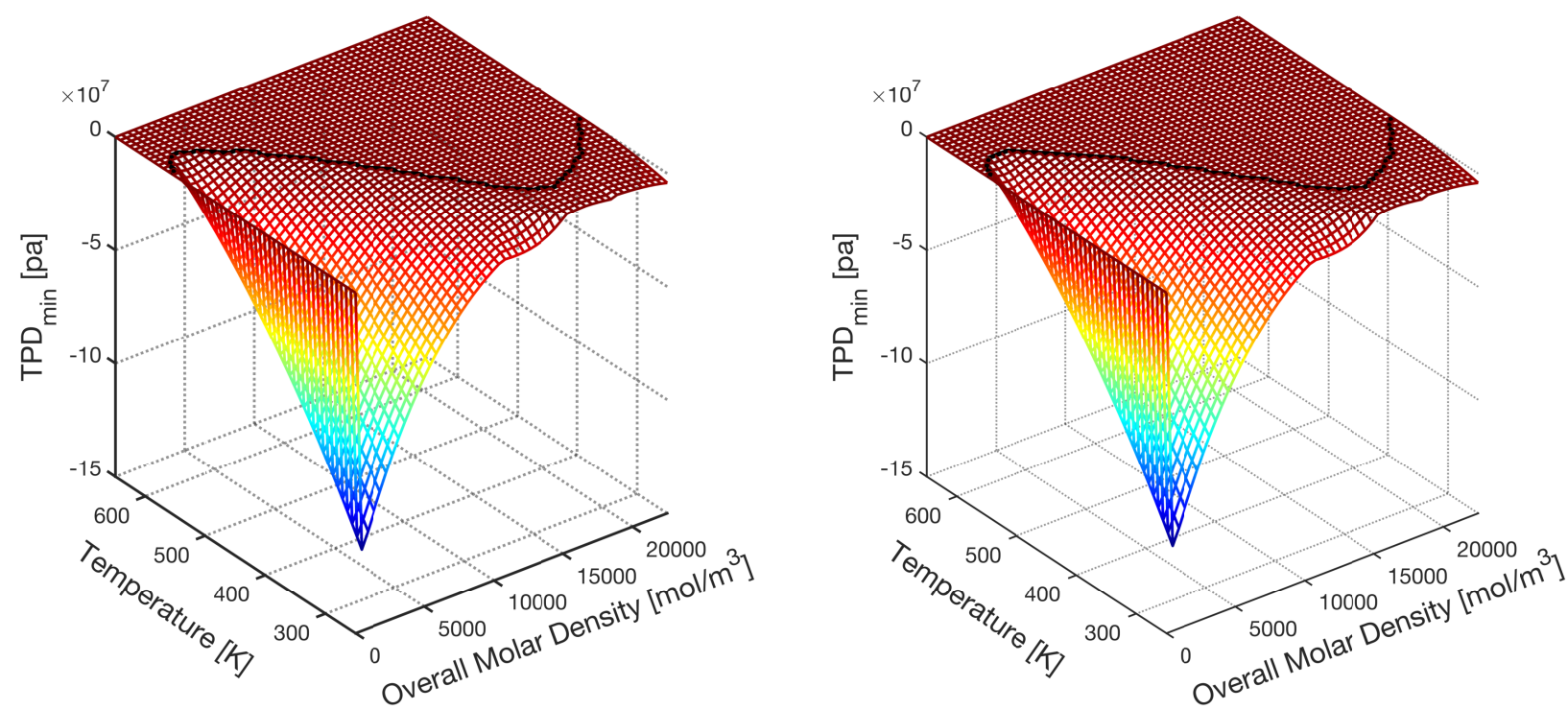

Figure 13: Global minimum of TPD function for the seven-component mixture of $\mathrm{N}_{2}, \mathrm{CO}_{2}$, $\mathrm{C}_{1}, \mathrm{PC}_{1}, \mathrm{PC}_{2}, \mathrm{PC}_{3}$ and $\mathrm{C}_{12+}$ in the presence of capillary pressure (left) and in the absence of capillary pressure (right), respectively. 


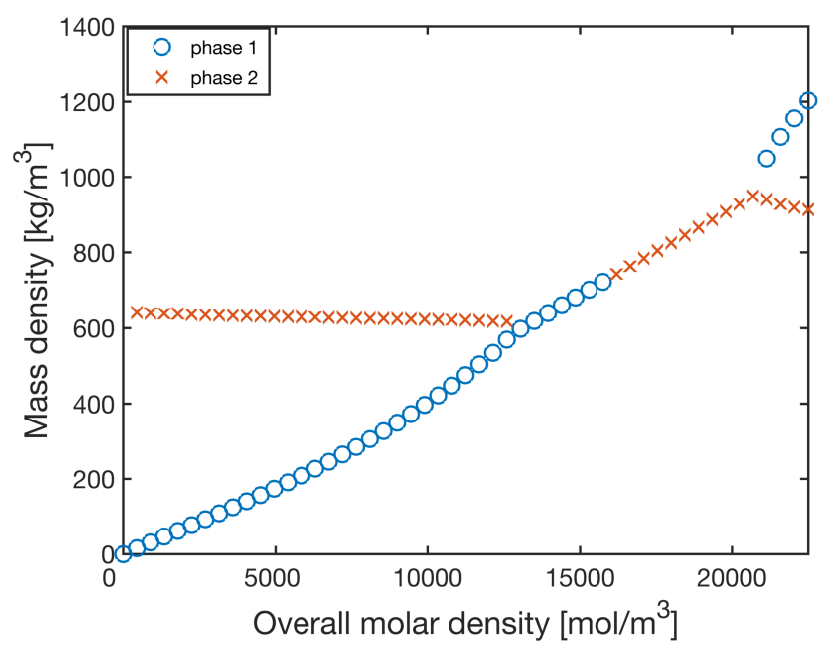

Figure 14: Mass density profile of two phases as a function of the overall molar density at $T=354 \mathrm{~K}$ in the absence of capillary pressure.

Compared to the unconfined phase equilibrium calculation, capillary compression results into an expanded vapor-liquid region at low overall molar density, implying the dew point curve expands outward to the single-vapor region. At intermediate molar density, the density difference between the vapor phase and the liquid phase diminishes so that the capillary effect is weakened. As a result, the confined fluid properties approach their bulk properties, and consequently the boundary between the single-liquid region and the vapor-liquid region has no change. In addition, it is observed the phase boundary of the liquid-liquid region is unaffected by the capillary pressue, which is attributed to the small density difference between two liquid phases. Moreover, the Weinaug-Katz correlation may not be suitable to compute the interfacial tension between two liquid phases. Figure 15 displays phase envelopes with and without capillary pressure. As discussed above, the dew point pressure variations take place at the tip of the phase envelope marked by the red circle. It can be seen in the inset figure that the dewpoint curve is further extended with the smaller pore radius. Furthermore, the occurance of the liquid-liquid region is accompanied by skyrocketing pressure change in the phase envelope. Figure 16 presents saturation and mole fraction of phase 1 as a function of the overall molar density with and without capillary pressure. Mole fractions of all seven components in the two-phase regions of phase 1 and phase 2 are compared in Figure 17 and 
Figure 18 respectively.

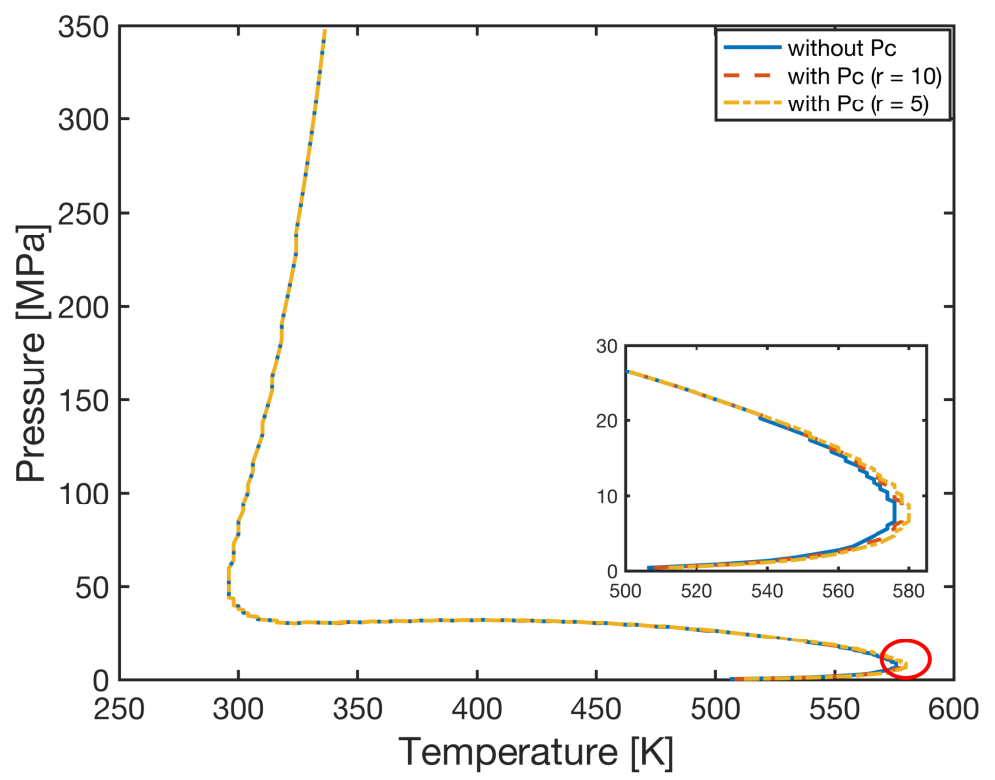

Figure 15: Phase envelope for the seven-component mixture of $\mathrm{N}_{2}, \mathrm{CO}_{2}, \mathrm{C}_{1}, \mathrm{PC}_{1}, \mathrm{PC}_{2}$, $\mathrm{PC}_{3}$ and $\mathrm{C}_{12+}$ with and without capillary pressure. The inset figure zooms in the change of dewpoint curve at the tip of phase envelope.
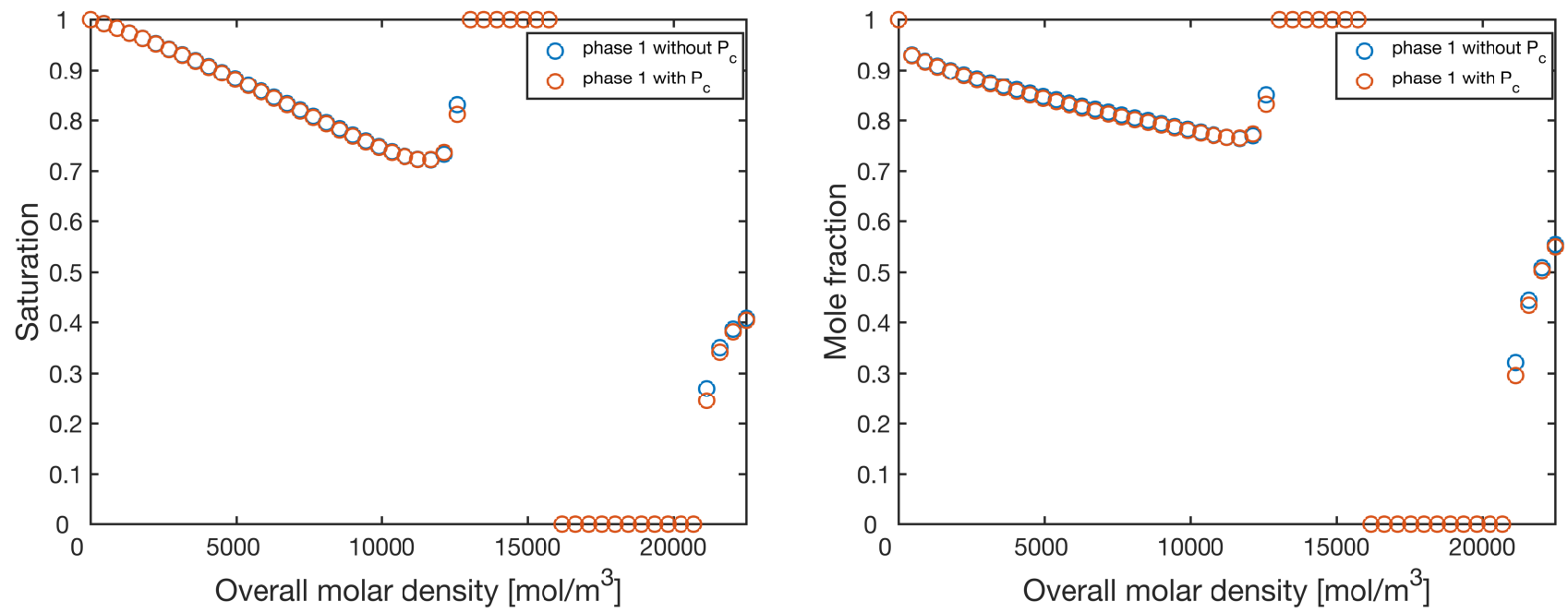

Figure 16: Saturation (left) and mole fraction (right) of phase 1 as a function of the overall molar density at $T=354 \mathrm{~K}$ with and without capillary pressure. 


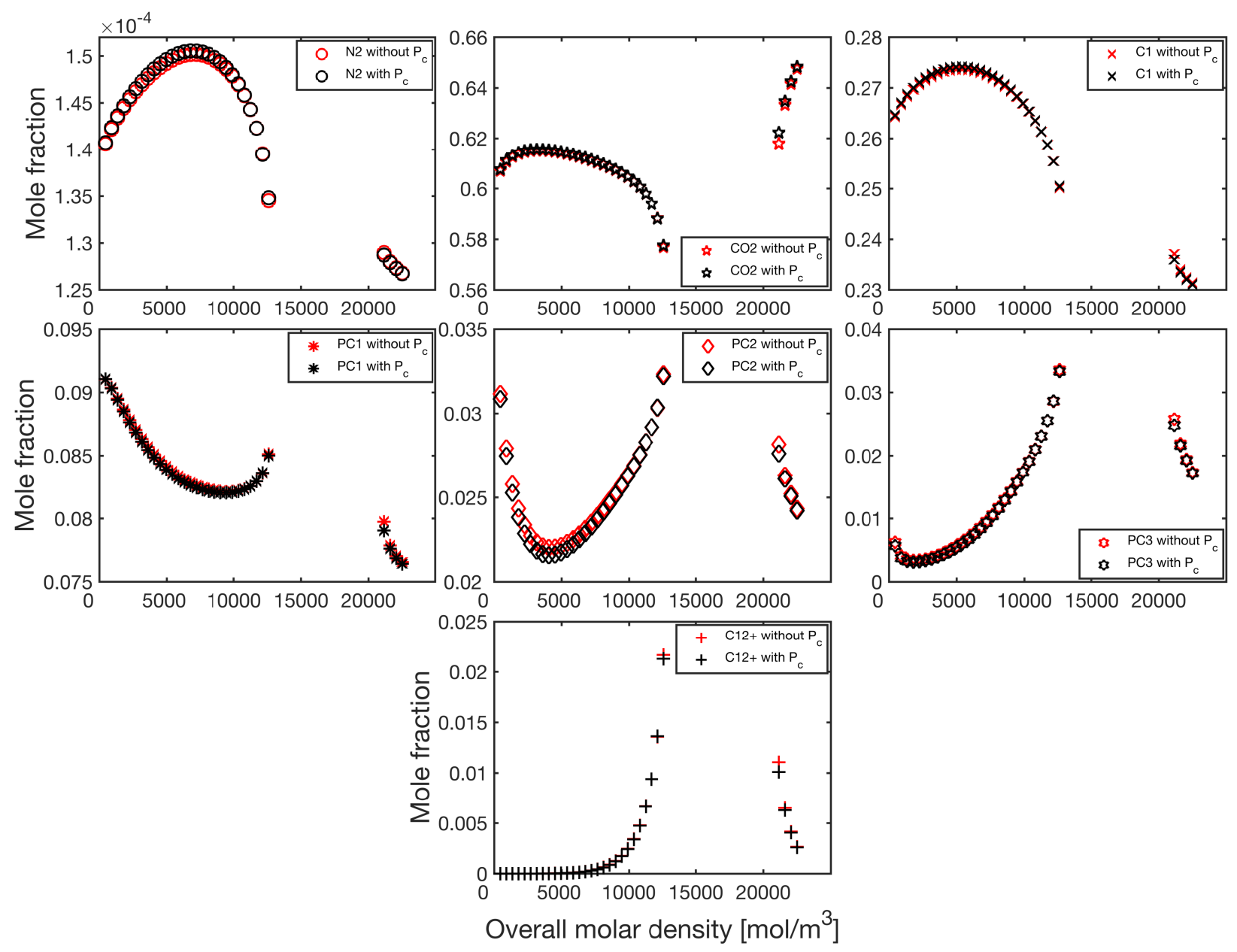

Figure 17: Mole fractions of all seven components in the two-phase regions of phase 1 as a function of the overall molar density at $T=354 \mathrm{~K}$ with and without capillary pressure. 

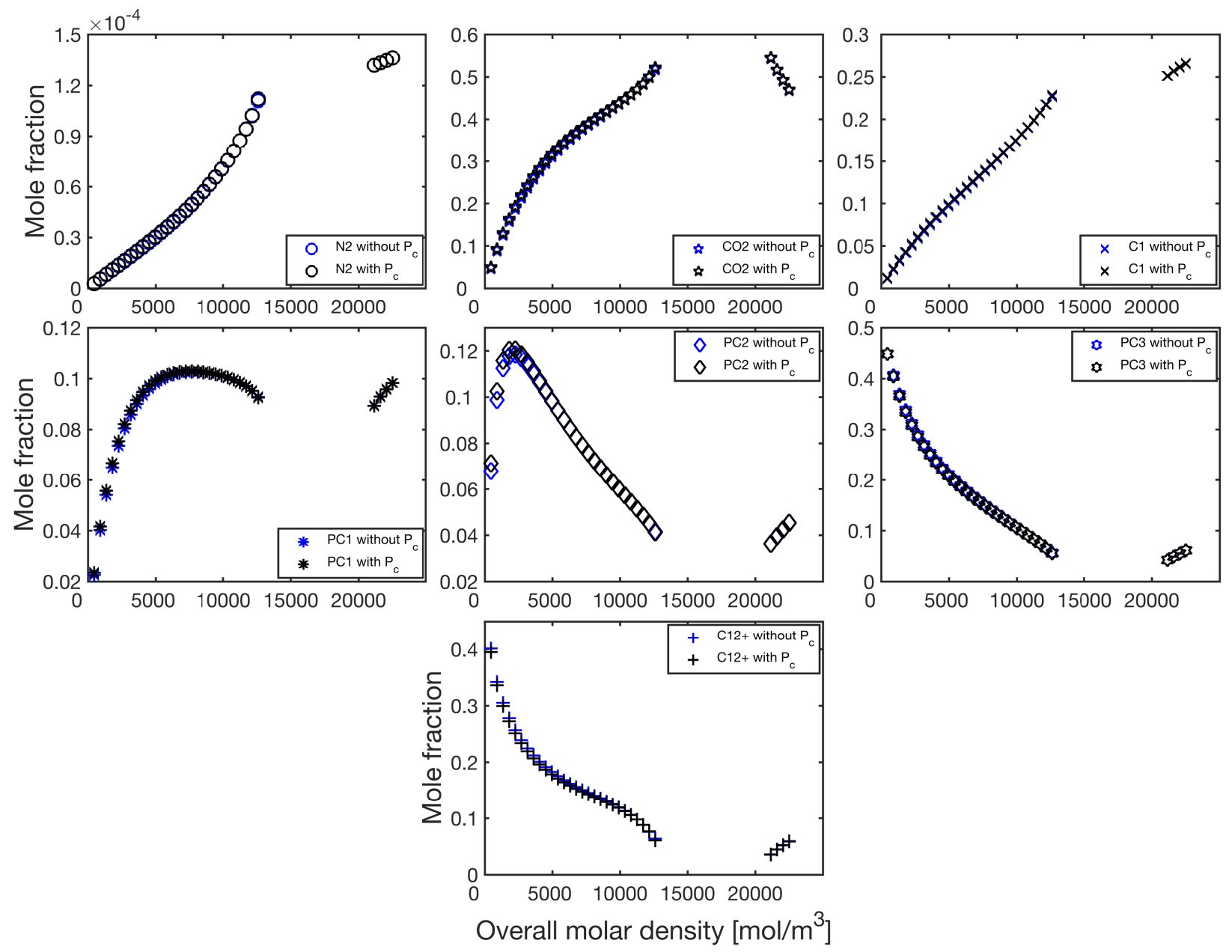

Figure 18: Mole fractions of all seven components in the two-phase regions of phase 2 as a function of the overall molar density at $T=354 \mathrm{~K}$ with and without capillary pressure.

\section{Conclusions}

In this study, the confined phase equilibrium calculation is performed by taking into account the capillary effect. Mole and volume evolution equations are developed to characterize the dynamic process from any non-equilibrium state to the equilibrium state in the presence of capillary pressure. A thermodynamically stable numerical algorithm is proposed to simultaneously solve the evolution equations with higher efficiency and accuracy. The robust performance of the proposed method is tested by a number of examples ranging from single-, binary- to multi-component fluid mixtures. When the capillary pressure is negligible, the 
proposed method degenerates to the unconfined VT-flash calculation. The computational results are highly consistent to the commonly used examples in published references.

Capillary effect reshapes the phase envelope. Even though we do not compute the exact critical point, the approximation solution gives quite close critical pressure and temperature at different pore radii, indirectly suggesting capillary pressure does not change the critical point. At high overall concentration, the bubble point pressure is suppressed in the presence

of capillary pressure, leading to the phase boundary contraction between the single-liquid region and vapor-liquid region. At low overall concentration, the expansion of dew point curve results in the phase boundary expanding outward to the single-vapor region. This agrees with the observation in published references that the dew point pressures increase in the upper branch of dew point curve while decrease in the lower branch.

It is worth noting that a single pore size and wettability are studied here. However, in reality, the reservoir rocks have complex pore size distribution and surface chemical heterogeneities. Thus, reservoir fluid in the nanopores has much more complicated phase behavior. As the pore size becomes smaller, the confinement effect has to be taken into account. Furthermore, adsorption and pore size reduction also play an important role in accurate estimation of the confined fluid properties. At some conditions, the fluid mixture may split into two liquid phases. The commonly used correlation to estimate the interfacial tension between vapor and liquid phases is possibly not suitable. Thus, an interfacial tension model involving the interaction between liquid and liquid phases could further extend the applicability of the current confined phase equilibrium calculation.

\section{Acknowledgement}

The authors gratefully acknowledge that the research reported in this publication was supported by funding from King Abdullah University of Science and Technology (KAUST) through the grants BAS/1/1351-01, URF/1/2993-01, and REP/1/2879-01 


\section{Supporting Information Available}

The proof of thermodynamically stable numerical algorithm.

This material is available free of charge via the Internet at http://pubs.acs.org/.

\section{References}

(1) Javadpour, F.; Fisher, D.; Unsworth, M. Nanoscale gas flow in shale gas sediments. J. Can. Petrol. Technol. 2007, 46, 55-61.

(2) Javadpour, F. Nanopores and apparent permeability of gas flow in mudrocks (shales and siltstone). J. Can. Petrol. Technol. 2009, 48, 16-21.

(3) Kuila, U.; Prasad, M. Specific surface area and pore-size distribution in clays and shales. Geophys. Prospect. 2013, 61, 341-362.

(4) Singh, S. K.; Sinha, A.; Deo, G.; Singh, J. K. Vapor-liquid phase coexistence, critical properties, and surface tension of confined alkanes. J. Phys. Chem. C 2009, 113, 71707180.

(5) Singh, S. K.; Singh, J. K. Effect of pore morphology on vapor-liquid phase transition and crossover behavior of critical properties from 3D to 2D. Fluid Phase Equilib. 2011, 300, 182-187.

(6) Zarragoicoechea, G. J.; Kuz, V. A. Critical shift of a confined fluid in a nanopore. Fluid Phase Equilib. 2004, 220, 7-9.

(7) Travalloni, L.; Castier, M.; W.Tavares, F.; Sandler, S. I. Critical behavior of pure confined fluids from an extension of the van der Waals equation of state. J. Supercrit. Fluids 2010, 55, 455-461. 
(8) Dong, X.; Liu, H.; Hou, J.; Wu, K.; Chen, Z. Phase Equilibria of Confined Fluids in Nanopores of Tight and Shale Rocks Considering the Effect of Capillary Pressure and Adsorption Film. Ind. Eng. Chem. Res. 2016, 55, 798-811.

(9) Brusilovsky, A. I. Mathematical simulation of phase behavior of natural multicomponent systems at high pressures with an equation of state. SPE Reservoir Eng. 1992, 7, $117-122$.

(10) Nojabaei, B.; Johns, R. T.; Chu, L. Effect of capillary pressure on phase behavior in tight rocks and shales. SPE Reservoir Eval. Eng. 2013, 16, 281-289.

(11) Peng, D. Y.; Robinson, D. B. A new two-constant equation of state. Ind. Eng. Chem. Fundam. 1976, 15, 59-64.

(12) Sandoval, D. R.; Yan, W.; Michelsen, M. L.; Stenby, E. H. The phase envelope of multicomponent mixtures in the presence of a capillary pressure difference. Ind. Eng. Chem. Res. 2016, 55, 6530-6538.

(13) Jin, L.; Ma, Y.; Jamili, A. Investigating the Effect of Pore Proximity on Phase Behavior and Fluid Properties in Shale Formations. SPE Annual Technical Conference and Exhibition. New Orleans, LA, 2013.

(14) Teklu, T. W.; Alharthy, N.; Kazemi, H.; Yin, X.; Graves, R. M.; AlSumaiti, A. M. Phase Behavior and Minimum Miscibility Pressure in Nanopores. SPE Reservoir Eval. Eng. 2014, 17, 396-403.

(15) Siripatrachai, N.; Ertekin, T.; Johns, R. T. Compositional simulation of discrete fractures incorporating the effect of capillary pressure on phase behavior. SPE Improved Oil Recovery Conference. Tulsa, OK, Apr 11 - 13, 2016.

(16) Wang, Y.; Yan, B.; Killough, J. Compositional modeling of tight oil using dynamic 
nanopore properties. SPE Annual Technical Conference and Exhibition. New Orleans, LA, Sep 29 - Oct 2, 2013.

(17) Rezaveisi, M.; Sepehrnoori, K.; Pope, G. A.; Johns, R. T. Compositional simulation including effect of capillary pressure on phase behavior. SPE Annual Technical Conference and Exhibition. Houston, TX, Sep 28 - 30, 2015.

(18) Mikyška, J.; Firoozabadi, A. A new thermodynamic function for phase-splitting at constant temperature, moles, and volume. AIChE J. 2011, 57, 1897-1904.

(19) Jindrová, T.; Mikyška, J. Fast and robust algorithm for calculation of two-phase equilibria at given volume, temperature, and moles. Fluid Phase Equilib. 2013, 353, 101-114.

(20) Jindrová, T.; Mikyška, J. General algorithm for multiphase equilibria calculation at given volume, temperature, and moles. Fluid Phase Equilib. 2015, 393, 7-25.

(21) Kou, J.; Sun, S.; Wang, X. An energy stable evolution method for simulating twophase equilibria of multi-component fluids at constant moles, volume and temperature. Comput. Geosci. 2016, 20, 283-295.

(22) Cabral, V. F.; Castier, M.; W.Tavares, F. Thermodynamic equilibrium in systems with multiple adsorbed and bulk phases. Chem. Eng. Sci. 2005, 60, 1773-1782.

(23) Smejkal, T.; Mikyška, J. Unified presentation and comparison of various formulations of the phase stability and phase equilibrium calculation problems. Fluid Phase Equilib. 2018

(24) Souza, A. T.; Cardozo-Filho, L.; Wolff, F.; Guirardello, R. Application of interval analysis for Gibbs and Helmholtz free energy global minimization in phase stability analysis. Braz. J. Chem. Eng. 2006, 23, 117-124.

(25) Mikyška, J.; Firoozabadi, A. Investigation of mixture stability at given volume, temperature, and number of moles. Fluid Phase Equilib. 2012, 321, 1-9. 
(26) Castier, M. Helmholtz function-based global phase stability test and its link to the isothermal-isochoric flash problem. Fluid Phase Equilib. 2014, 379, 104-111.

(27) Nichita, D. V.; de los Angeles Duran Valencia, C.; Gomez, S. Volume-based thermodynamics global phase stability analysis. Chem. Eng. Commun. 2006, 193, 1194-1216.

(28) Nichita, D. V. Fast and robust phase stability testing at isothermal-isochoric conditions. Fluid Phase Equilib. 2017, 447, 107-124.

(29) Nagarajan, N. R.; Cullick, A. S.; Griewank, A. New strategy for phase equilibrium and critical point calculations by thermodynamic energy analysis. Part I. Stability analysis and flash. Fluid Phase Equilib. 1991, 62, 191-210.

(30) Kou, J.; Sun, S. A stable algorithm for calculating phase equilibria with capillarity at specified moles, volume and temperature using a dynamic model. Fluid Phase Equilib. 2018, 456, 7-24.

(31) Weinaug, C. F.; Katz, D. L. Surface tensions of methane-propane mixtures. Ind. Eng. Chem. Res. 1943, 35, 239-246.

(32) de Groot, S. R.; Mazur., P. Non-equilibrium thermodynamics; Courier Corporation, 2013.

(33) Kou, J.; Sun, S. Numerical methods for a multicomponent two-phase interface model with geometric mean influence parameters. SIAM J. Sci. Comput. 2015, 37, B543B569.

(34) Kou, J.; Sun, S.; Wang, X. Efficient numerical methods for simulating surface tension of multi-component mixtures with the gradient theory of fluid interfaces. Comput. Methods Appl. Mech. Eng. 2015, 292, 92-106. 
(35) Kou, J.; Sun, S. Unconditionally stable methods for simulating multi-component twophase interface models with Peng-Robinson equation of state and various boundary conditions. J. Comput. Appl. Math. 2016, 291, 158-182.

(36) Qiao, Z.; Sun, S. Two-phase fluid simulation using a diffuse interface model with PengRobinson equation of state. SIAM J. Sci. Comput. 2014, 36, B708-B728.

(37) Fan, X.; Kou, J.; Qiao, Z.; Sun, S. A Componentwise Convex Splitting Scheme for Diffuse Interface Models with Van der Waals and Peng-Robinson Equations of State. SIAM J. Sci. Comput. 2017, 39, B1-B28.

(38) Li, Y.; Kou, J.; Sun, S. Numerical modeling of isothermal compositional grading by convex splitting methods. J. Nat. Gas Sci. Eng. 2017, 43, 207-221.

(39) Schnabel, R. B.; Eskow, E. A revised modified Cholesky factorization algorithm. SIAM J. Optim. 1999, 9, 1135-1148.

(40) Firoozabadi, A.; Katz, D. L. Surface tension of reservoir crude oil/gas systems recognizing the asphalt in the heavy fraction. SPE Reservoir Eng. 1988, 3, 265-272. 


\section{Graphical TOC Entry}

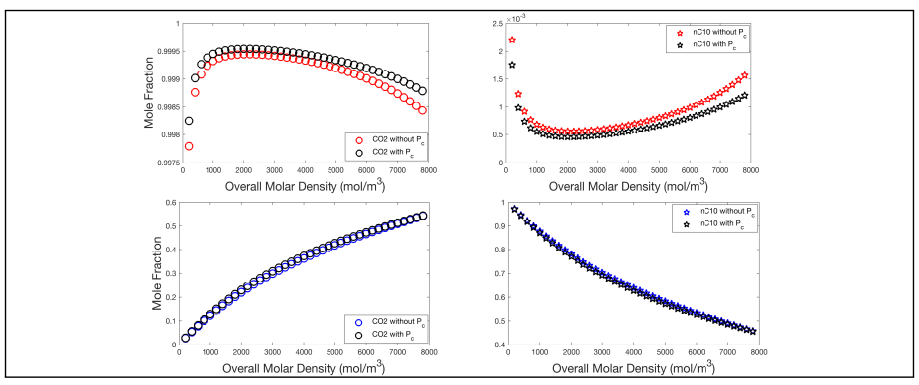

